\title{
New Estimates of the Nonlinear Fourier Transform for the Defocusing NLS Equation
}

\section{Jan-Cornelius Molnar}

Institut für Mathematik, Universität Zürich, Winterthurerstrasse 190, CH-8057 Zürich, Switzerland

Correspondence to be sent to: jan.molnar@math.uzh.ch

The defocusing NLS equation $\mathrm{i} u_{t}=-u_{x x}+2|u|^{2} u$ on the circle admits a global nonlinear Fourier transform, also known as Birkhoff map, linearizing the NLS flow. The regularity properties of $u$ are known to be closely related to the decay properties of the corresponding nonlinear Fourier coefficients. In this paper, we quantify this relationship by providing two-sided polynomial estimates of all integer Sobolev norms $\|u\|_{m}, m \geqslant 0$, in terms of the weighted norms of the nonlinear Fourier transformed.

\section{Introduction}

Consider the defocusing nonlinear Schrödinger (NLS) equation

$$
\mathrm{i} \partial_{t} u=-\partial_{x}^{2} u+2|u|^{2} u, \quad x \in \mathbb{T},
$$

on the circle $\mathbb{T}=\mathbb{R} / \mathbb{Z}$ with $u$ taken from $L^{2}:=L^{2}(\mathbb{T}, \mathbb{C})$. As is well known, the NLS equation can be written as an infinite-dimensional Hamiltonian system. We introduce the phase space $L_{c}^{2}:=L^{2} \times L^{2}$ with elements $\varphi=\left(\varphi_{-}, \varphi_{+}\right)$and define the inner product

$$
\langle\varphi, \psi\rangle:=\int_{\mathbb{T}} \varphi_{+} \bar{\psi}_{+}+\varphi_{-} \bar{\psi}_{-} \mathrm{d} x
$$

Received April 3, 2014; Accepted October 14, 2014

Communicated by Lenells, Jonatan

(C) The Author(s) 2014. Published by Oxford University Press. All rights reserved. For permissions, please e-mail: journals.permissions@oup.com. 
which makes $L_{c}^{2}$ a Hilbert space. The associated norm is denoted by $\|\varphi\|_{0}$. Further, we endow this space with the Poisson structure given by

$$
\{F, G\}:=-\mathrm{i} \int_{\mathbb{T}}\left(\partial_{\varphi_{-}} F \partial_{\varphi_{+}} G-\partial_{\varphi_{+}} F \partial_{\varphi_{-}} G\right) \mathrm{d} x .
$$

Here $\partial_{\varphi_{-}} F$ and $\partial_{\varphi_{+}} F$ denote the components of the $L^{2}$-gradient $\partial_{\varphi} F$ of a $C^{1}$-functional $F$. The Hamiltonian system with Hamiltonian

$$
\mathcal{H}\left(\varphi_{-}, \varphi_{+}\right):=\int_{\mathbb{T}}\left(\partial_{X} \varphi_{-} \partial_{X} \varphi_{+}+\varphi_{-}^{2} \varphi_{+}^{2}\right) \mathrm{d} x
$$

is then given by

$$
\mathrm{i} \partial_{t}\left(\varphi_{-}, \varphi_{+}\right)=\left(\partial_{\varphi_{+}} \mathcal{H},-\partial_{\varphi_{-}} \mathcal{H}\right)
$$

and the defocusing NLS is obtained by restriction to the invariant subspace of real-type states

$$
L_{r}^{2}:=\left\{\varphi \in L_{c}^{2}: \varphi^{*}=\varphi\right\}, \quad \varphi^{*}=\left(\overline{\varphi_{+}}, \overline{\varphi_{-}}\right) .
$$

Indeed, with $\varphi=(u, \bar{u})$ the defocusing NLS equation becomes

$$
\mathrm{i} \partial_{t} u=\mathrm{i}\{u, \mathcal{H}\}=\partial_{\bar{u}} \mathcal{H}(u, \bar{u}) .
$$

After the KdV equation, the NLS equation was the second evolution equation known to be completely integrable by the inverse scattering method [27]. In fact, according to [10] (cf. also [1, 2, 23]), the defocusing NLS equation is integrable in the strongest possible sense meaning that it admits global Birkhoff coordinates $\left(x_{n}, y_{n}\right)_{n \in \mathbb{Z}}$.

To state our main result, let $\sum_{n \in \mathbb{Z}}\left(\varphi_{2 n}^{-} \mathrm{e}^{-\mathrm{i} 2 n \pi x}, \varphi_{2 n}^{+} \mathrm{e}^{\mathrm{i} 2 n \pi x}\right)$ denote the Fourier series of $\varphi=\left(\varphi_{-}, \varphi_{+}\right) \in L_{c}^{2}$ and introduce for any $s \geqslant 0$ the Sobolev norm $\|\varphi\|_{s}$ given by

$$
\|\varphi\|_{s}^{2}:=\sum_{n \in \mathbb{Z}}\langle 2 n \pi\rangle^{2 s}\left(\left|\varphi_{2 n}^{-}\right|^{2}+\left|\varphi_{2 n}^{+}\right|^{2}\right), \quad\langle x\rangle:=1+|x| .
$$

The space of all $\varphi \in L_{c}^{2}$ with $\|\varphi\|_{s}<\infty$ is denoted by $H_{c}^{s}$, and $H_{r}^{s}:=L_{r}^{2} \cap H_{c}^{s}$. Furthermore, let us introduce the model space

$$
h_{r}^{s}:=\left\{(x, y)=\left(x_{n}, y_{n}\right)_{n \in \mathbb{Z}}:\|(x, y)\|_{s}:=\left(\|x\|_{s}^{2}+\|y\|_{s}^{2}\right)^{1 / 2}<\infty\right\},
$$

where the norm $\|x\|_{s}$ is defined by

$$
\|x\|_{s}^{2}:=\sum_{n \in \mathbb{Z}}\langle 2 n \pi\rangle^{2 s}\left|x_{n}\right|^{2}
$$


This space is endowed with the canonical Poisson structure $\left\{X_{n}, Y_{m}\right\}=-\delta_{n, m}$ while all other brackets vanish.

The Birkhoff map $\varphi \mapsto\left(x_{n}, Y_{n}\right)_{n \in \mathbb{Z}}$ is a bi-analytic, canonical diffeomorphism $\Omega: H_{r}^{0} \rightarrow h_{r}^{0}$, whose restriction $\Omega: H_{r}^{m} \rightarrow h_{r}^{m}$ is again bi-analytic for any integer $m \geqslant 1$. On $h_{r}^{1}$ the transformed NLS Hamiltonian $\mathcal{H} \circ \Omega^{-1}$ is a real analytic function of the actions $I_{n}=\left(x_{n}^{2}+Y_{n}^{2}\right) / 2$ alone and the NLS evolution takes the particularly simple form

$$
\dot{x}_{n}=-\omega_{n} Y_{n}, \quad \dot{Y}_{n}=\omega_{n} X_{n}, \quad \omega_{n}:=\partial_{I_{n}} \mathcal{H} .
$$

One may thus think of $\Omega$ as a nonlinear Fourier transform for the defocusing NLS equation. Remarkably, the derivative $\mathrm{d}_{0} \Omega$ of $\Omega$ at the origin is the Fourier transform and on $L_{r}^{2}$, as for the Fourier transform,

$$
\|\Omega(\varphi)\|_{0}=\|\varphi\|_{0},
$$

which we also refer to as Parseval's identity—cf., for example, [10, 23]. Our main result says that for higher order Sobolev norms the following version of Parseval's identity holds for the nonlinear map $\Omega$.

Theorem 1.1. For any integer $m \geqslant 1$, there exist absolute constants $c_{m}, d_{m}>0$ such that the restriction of $\Omega$ to $H_{r}^{m}$ satisfies the two-sided estimates

$$
\text { (i) }\|\Omega(\varphi)\|_{m} \leqslant c_{m}\left(\|\varphi\|_{m}+\left(1+\|\varphi\|_{\tilde{m}-1}\right)^{2 m}\|\varphi\|_{\tilde{m}-1}\right) \text {, }
$$

where $\tilde{m}=\max (m, 2)$, and

$$
\text { (ii) }\|\varphi\|_{m} \leqslant d_{m}\left(\|\Omega(\varphi)\|_{m}+\left(1+\|\Omega(\varphi)\|_{m-1}\right)^{4 m-3}\|\Omega(\varphi)\|_{m-1}\right) .
$$

The main feature of Theorem 1.1 is that the constants $c_{m}$ and $b_{m}$ can be chosen independently of $\varphi$.

Note that the estimate (i) is linear in the highest Sobolev norm $\|\varphi\|_{m}$ for $m \geqslant 2$, and that the estimate (ii) is linear in the highest weighted $h_{r}^{m}$-norm $\|\Omega(\varphi)\|_{m}$ of $\Omega(\varphi)$. Hence, Theorem 1.1 shows that the 1-smoothing property of the Birkhoff map $\Omega$ established in [17] holds in a uniform fashion.

The proof of Theorem 1.1 relies on estimates of the action variables $I(\varphi)=\left(I_{n}\right)_{n \in \mathbb{Z}}$ where $I_{n}=\left(x_{n}^{2}+y_{n}^{2}\right) / 2, n \in \mathbb{Z}$. The decay properties of the actions $I_{n}$ are known to be closely related to the regularity properties of $\varphi-c f .[7,15,16]$. We need to quantify this relationship by providing two-sided estimates of the Sobolev norms of $\varphi$ in terms of weighted $\ell^{1}$-norms of $I(\varphi)$. For that purpose introduce the weighted sequence space 
$\ell_{s}^{1}$ whose norm is defined by

$$
\|I(\varphi)\|_{\ell_{s}^{1}}:=\sum_{n \in \mathbb{Z}}\langle 2 n \pi\rangle^{s}\left|I_{n}(\varphi)\right| .
$$

Theorem 1.2. For any integer $m \geqslant 1$, there exist absolute constants $c_{m}$ and $d_{m}$, such that for all $\varphi \in H_{r}^{m}$

$$
\text { (i) } \quad\|I(\varphi)\|_{\ell_{2 m}^{1}} \leqslant c_{m}^{2}\left(\|\varphi\|_{m}^{2}+\left(1+\|\varphi\|_{\tilde{m}-1}\right)^{4 m}\|\varphi\|_{\tilde{m}-1}^{2}\right) \text {, }
$$

where $\tilde{m}=\max (m, 2)$, and

$$
\text { (ii) }\|\varphi\|_{m}^{2} \leqslant d_{m}^{2}\left(\|I(\varphi)\|_{\ell_{2 m}^{1}}+\left(1+\|I(\varphi)\|_{\ell_{2 m-2}^{1}}\right)^{4 m-3}\|I(\varphi)\|_{\ell_{2 m-2}^{1}}\right) .
$$

Remark. The same constants $c_{m}, d_{m}$ of Theorem 1.1 can be used in Theorem 1.2.

It turns out that versions of the estimates (i) of Theorems 1.1 and 1.2 hold for a larger family of spaces referred to weighted Sobolev spaces-see [12,13] for an introduction. A normalized, submultiplicative, and monotone weight is a symmetric function $w: \mathbb{Z} \rightarrow \mathbb{R}$ with

$$
w_{n} \geqslant 1, \quad w_{n}=w_{-n}, \quad w_{n+m} \leqslant w_{n} w_{m}, \quad w_{|n|} \leqslant w_{|n|+1},
$$

for all $n, m \in \mathbb{Z}$. The class of all such weights is denoted by $\mathcal{M}$ and $H_{c}^{w}$ is the space of $L_{c}^{2}$ functions $\varphi$ with finite $w$-norm

$$
\|\varphi\|_{w}^{2}:=\sum_{n \in \mathbb{Z}} w_{2 n}^{2}\left(\left|\varphi_{2 n}^{-}\right|^{2}+\left|\varphi_{2 n}^{+}\right|^{2}\right) .
$$

Furthermore, $h_{r}^{w}$ denotes the subspace of $\ell_{r}^{2}$ where $\|x\|_{w}^{2}+\|y\|_{w}^{2}<\infty$,

$$
\|x\|_{w}^{2}:=\sum_{n \in \mathbb{Z}} w_{2 n}^{2}\left|x_{n}\right|^{2} .
$$

For any $s \geqslant 0$, the Sobolev weight $\langle n \pi\rangle^{s}$ gives rise to the usual Sobolev space $H_{c}^{s}$. For $s \geqslant 0$ and $a>0$, the Abel weight $\langle n \pi\rangle^{s} \mathrm{e}^{a|n|}$ gives rise to the space $H_{c}^{s, a}$ of $L_{c}^{2}$-functions, which can be analytically extended to the open strip $\{z:|\Im z|<a / 2 \pi\}$ of the complex plane with traces in $H_{c}^{s}$ on the boundary lines. In between are, among others, the Gevrey weights

$$
\langle n\rangle^{s} \mathrm{e}^{a|n|^{\sigma}}, \quad 0<\sigma<1, s \geqslant 0, a>0
$$

which give rise to the Gevrey spaces $H_{c}^{s, a, \sigma}$, as well as weights of the form

$$
\langle n\rangle^{s} \exp \left(\frac{a|n|}{1+\log ^{\sigma}\langle n\rangle}\right), \quad 0<\sigma<1, s \geqslant 0, a>0,
$$

that are lighter than Abel weights but heavier than Gevrey weights. 
To avoid certain technicalities in our estimates, we restrict ourselves to weights incorporating a factor which grows at a linear rate. We thus introduce the subclass

$$
\mathcal{M}^{1}:=\left\{w \in \mathcal{M}: w_{n}=\langle n\rangle v_{n} \text { for all } n \in \mathbb{Z} \text { with some } v \in \mathcal{M}\right\} .
$$

Finally, we assume that all weights $w \in \mathcal{M}$ are piecewise linearly extended to functions on the real line $w: \mathbb{R} \rightarrow \mathbb{R}_{>0}, t \mapsto w[t]$.

Theorem 1.3. For any weight $w \in \mathcal{M}^{1}$, there exists a complex neighborhood $W_{w}$ of $H_{r}^{w}$ within $H_{c}^{w}$ and a constant $c_{w}$, such that

$$
\text { (i) } \sum_{n \in \mathbb{Z}} w_{2 n}^{2}\left|I_{n}\right| \leqslant c_{w}^{2} w\left[16\|\varphi\|_{w}^{2}\right]^{2}\|\varphi\|_{w}^{2} .
$$

Moreover, the restriction of the Birkhoff map $\Omega$ to $H_{r}^{w}$ takes values in $h_{r}^{w}$ and satisfies

$$
\text { (ii) }\|\Omega(\varphi)\|_{w} \leqslant C_{w} w\left[16\|\varphi\|_{w}^{2}\right]\|\varphi\|_{w} \text {. }
$$

In this more general set up, the bounds in (i) and (ii) of Theorem 1.3 are of the same type as the weight, and are valid for all submultiplicative weights including those growing exponentially fast. The following version of Theorem 1.3 for Sobolev spaces of real exponent complements the results of Theorems 1.2 and 1.3 .

Corollary 1.4. For any real $s \geqslant 1$, there exist a complex neighborhood $W_{s}$ of $H_{r}^{s}$ and a constant $c_{s}$ such that

$$
\|I(\varphi)\|_{\ell_{2 s}^{1}} \leqslant c_{s}^{2}\left(1+\|\varphi\|_{s}\right)^{4 s}\|\varphi\|_{s}^{2} .
$$

Moreover, the restriction of the Birkhoff map $\Omega$ to $H_{r}^{s}$ takes values in $h_{r}^{s}$ and satisfies

$$
\|\Omega(\varphi)\|_{s} \leqslant C_{S}\left(1+\|\varphi\|_{s}\right)^{2 s}\|\varphi\|_{s}
$$

Outline. The action variables $I_{n}$ and more generally the action variables $J_{n, k}$ on levels $k \geqslant 1$ can be defined entirely in terms of the periodic spectrum of the associated Zakharov-Shabat operator used in the Lax-pair formulation of the NLS equation. More to the point, consider the operator

$$
L(\varphi)=\left(\begin{array}{cc}
\mathrm{i} & \\
& -\mathrm{i}
\end{array}\right) \frac{\mathrm{d}}{\mathrm{d} x}+\left(\begin{array}{ll} 
& \psi \\
\bar{\psi} &
\end{array}\right),
$$

with periodic boundary conditions on the interval $[0,2]$ of twice the length of the periodicity of $\varphi=(\psi, \bar{\psi}) \in L_{r}^{2}$. The spectrum of $L(\varphi)$ is well known to be real, pure point, and 
to consist of a double infinite sequence of eigenvalues

$$
\cdots \leqslant \lambda_{n-1}^{+}<\lambda_{n}^{-} \leqslant \lambda_{n}^{+}<\lambda_{n+1}^{-} \leqslant \cdots
$$

with asymptotic behavior $\lambda_{n}^{ \pm} \sim n \pi$ as $|n| \rightarrow \infty$. The asymptotic behavior of the actions on odd levels $k=2 m+1$ turns out to be

$$
J_{n, 2 m+1} \sim\left(\lambda_{n}^{ \pm}\right)^{2 m} I_{n} \sim(n \pi)^{2 m} I_{n}, \quad|n| \rightarrow \infty
$$

and they satisfy the trace formula

$$
\sum_{n \geqslant 1} J_{n, 2 m+1}=\frac{(-1)^{m+1}}{4^{m}} \mathcal{H}_{2 m+1}, \quad m \geqslant 1,
$$

where $\mathcal{H}_{2 m+1}$ denotes the $2 m+1$ th Hamiltonian in the NLS hierarchy,

$$
\mathcal{H}_{1}=\int_{\mathbb{T}}|\psi|^{2} \mathrm{~d} x, \quad \mathcal{H}_{3}=\int_{\mathbb{T}}\left(\left|\psi^{\prime}\right|^{2}+|\psi|^{4}\right) \mathrm{d} x, \ldots
$$

Note that $\mathcal{H}_{3}$ denotes the Hamiltonian of the NLS equation. On $H_{r}^{m}$, for $m \geqslant 1$,

$$
\mathcal{H}_{2 m+1}=(-1)^{(m+1)} \int_{\mathbb{T}}\left(\left|\psi^{(m)}\right|^{2}+p_{m}\left(\psi, \ldots, \psi^{(m-1)}\right)\right) \mathrm{d} x
$$

where $p_{m}$ is a polynomial expression in $\psi$ and its first $m-1$ derivatives. Viewing $\mathcal{H}_{2 m+1}$ as a lower order perturbation of the $H_{r}^{m}$-norm we formally obtain

$$
\sum_{n \in \mathbb{Z}}(n \pi)^{2 m} I_{n} \sim \sum_{n \in \mathbb{Z}} J_{n, 2 m+1} \sim\|\varphi\|_{m}^{2}+O\left(\|\varphi\|_{m-1}\right)
$$

The essential ingredient to make this formal statement explicit is a sufficiently accurate localization of the periodic eigenvalues $\lambda_{n}^{ \pm}$whose threshold in $|n|$ depends only on $\|\varphi\|_{1}$. For $|n|$ above this threshold, we can directly compare the weighted action norms and the polynomial expressions in $\varphi$ as described above, while the remainder for $|n|$ below the threshold can be regard as an $H^{1}$-error term. Thereof we obtain Theorem 1.2, which directly implies Theorem 1.1. Note that our method of proof completely avoids the use of auxiliary spectral quantities such as spectral heights or conformal mapping theory.

To prove Theorem 1.3, we take a slightly different approach by quantitatively estimating the action variables in terms of the spacing of the periodic eigenvalues of the associated Zakharov-Shabat operator. For the latter, we obtain estimates in any weighted norm, which allows us to obtain Theorem 1.3. 
Related results. Theorem 1.1 for $m=0$ is referred to as Parseval's identity,

$$
\frac{1}{2}\|\Omega(\varphi)\|_{0}=\|I(\varphi)\|_{\ell^{1}}=\frac{1}{2}\|\varphi\|_{0}^{2},
$$

and is well known-see [10, 23]. The case $m=1$ was proved by Korotyaev [19] using conformal mapping theory, see also [21]. However, his method does not seem applicable for the case $m \geqslant 2$. In fact, it is stated as an open problem in [21].

For the case of the $\mathrm{KdV}$ equation

$$
\partial_{t} u=-\partial_{x}^{3} u+6 u \partial_{x} u, \quad x \in \mathbb{T},
$$

Korotyaev $[18,20]$ obtained polynomial bounds of the Sobolev norms $\|u\|_{m}$ in terms of the action variables where the order of the polynomials grows factorial in $m$. Note that the bound in (ii) of Theorem 1.2 is of order 1 in the Sobolev norm $\|\varphi\|_{m}$ and the order of the remainder grows linearly in $m$. It turns out that our method can also be applied to the KdV equation. In [25], we improve on the bounds obtained by Korotyaev in [18, 20].

For NLS in weighted Sobolev spaces, the qualitative relationship

$$
\varphi \in H_{r}^{w} \Leftrightarrow \Omega(\varphi) \in h_{r}^{w}
$$

is a corollary of the methods presented in $[7,16]$ —at least for weights growing at subexponential speed. To the best of our knowledge, the estimate of $\|\Omega(\varphi)\|_{w}$ on $H_{r}^{w}$ as well as the estimate of $\|I(\varphi)\|_{w}$ on a small complex neighborhood of $H_{r}^{w}$ as presented in Theorem 1.3 are new.

Viewing the action $I_{n}$ as a 1-smoothing perturbation of the squared modulus of the nth Fourier coefficient, our method of comparing the weighted action norms with the Hamiltonians of the NLS hierarchy amounts to a separate analysis of Fourier modes of low and high frequencies. This idea has a long history in the analysis of nonlinear partial differential equations. Most recently, it lead Colliander et al. [3-6] to invent the I-Method, which allows to obtain global well posedness of subcritical equations in low regularity regimes where the Hamiltonian (or other integrals) of the equation cease to be well defined. The idea is to damp all sufficiently high Fourier modes of a local solution such that the Hamiltonian can be controlled by weaker norms while still being an almost conserved quantity. The difficulty here is to choose the damping subtle enough such that the nonlinearity of the equation does not create a significant interaction of low and high frequencies. Our situation is so to say opposed to that of the I-Method: As we aim for quantitative global estimates, controlling the modes of low frequencies is the most delicate part. Here the localization of the periodic eigenvalues of the Zakharov-Shabat 
operator associated with the NLS equation plays a crucial role. Note that there exists a vast amount of literature on the spectral theory of these operators-see, for example, $[7,10,11,13,21]$ and the references therein.

Organization of the paper. In Section 2, we recall the definition of the NLS action variables on integer levels $k \geqslant 1$ as well as the trace formulae relating them to the hierarchy of NLS Hamiltonians. The somewhat lengthy proof of the analyticity properties of the action integrand can be found in Appendix 1. The quadratic localization of the Zakharov-Shabat spectrum is obtained in Section 3, and is subsequently used in Sections 4 and 5 to obtain Theorem 1.2(i) and (ii), respectively. In Section 6, we obtain the estimate of the actions in terms of the spacing of the Zakharov-Shabat eigenvalues which implies Theorem 1.3.

\section{Setup}

In this section, we briefly recall the definition of NLS action variables from [10], as well as the main properties of the periodic spectrum of Zakharov-Shabat operators used to define them.

For a potential $\varphi=\left(\varphi_{-}, \varphi_{+}\right) \in H_{c}^{0}=L_{c}^{2}$, consider the Zakharov-Shabat operator

$$
L(\varphi):=\left(\begin{array}{ll}
\mathrm{i} & \\
& -\mathrm{i}
\end{array}\right) \frac{\mathrm{d}}{\mathrm{d} x}+\left(\begin{array}{ll} 
& \varphi_{-} \\
\varphi_{+} &
\end{array}\right)
$$

on the interval $[0,2]$ with periodic boundary conditions. The spectrum of $L(\varphi)$ is well known to be pure point, and more precisely, to consist of a sequence of pairs of complex eigenvalues $\lambda_{n}^{+}(\varphi)$ and $\lambda_{n}^{-}(\varphi)$, listed with algebraic multiplicities, such that

$$
\lambda_{n}^{ \pm}(\varphi)=n \pi+\ell_{n}^{2}, \quad n \in \mathbb{Z}
$$

Here $\ell_{n}^{2}$ denotes a generic $\ell^{2}$-sequence. We may order the eigenvalues lexicographicallyfirst by their real part, and second by their imaginary part-to represented them as a bi-infinite sequence of complex eigenvalues

$$
\cdots \preccurlyeq \lambda_{n-1}^{+} \preccurlyeq \lambda_{n}^{-} \preccurlyeq \lambda_{n}^{+} \preccurlyeq \lambda_{n+1}^{-} \preccurlyeq \cdots
$$

By a slight abuse of notation, we call the eigenvalues of $L(\varphi)$ the periodic spectrum of $\varphi$. Further we introduce the gap lengths

$$
\gamma_{n}:=\lambda_{n}^{+}-\lambda_{n}^{-}=\ell_{n}^{2}, \quad n \in \mathbb{Z}
$$


To obtain another characterization of the periodic spectrum, we denote by $M(x, \lambda, \varphi)$ the standard fundamental solution of the ordinary differential equation $L(\varphi) M=\lambda M$, and introduce the discriminant

$$
\Delta(\lambda, \varphi):=\operatorname{tr} M(1, \lambda, \varphi)
$$

To simplify matters, we may drop some or all of its arguments from the notation, whenever there is no danger of confusion. The periodic spectrum of $\varphi$ is precisely the zero set of the entire function $\Delta^{2}(\lambda)-4$, and we have the product representation

$$
\Delta^{2}(\lambda)-4=-4 \prod_{n \in \mathbb{Z}} \frac{\left(\lambda_{n}^{+}-\lambda\right)\left(\lambda_{n}^{-}-\lambda\right)}{\pi_{n}^{2}}, \quad \pi_{n}:= \begin{cases}n \pi, & n \neq 0 \\ 1, & n=0 .\end{cases}
$$

Hence, this function is a spectral invariant. We also need the $\lambda$-derivative $\Delta^{\bullet}:=\partial_{\lambda} \Delta$ whose zeros are denoted by $\lambda_{n}^{\bullet}$ and satisfy $\lambda_{n}^{\bullet}=n \pi+\ell_{n}^{2}$. This derivative has the product representation

$$
\Delta^{\bullet}(\lambda)=2 \prod_{n \in \mathbb{Z}} \frac{\lambda_{n}^{\bullet}-\lambda}{\pi_{n}}
$$

If the potential $\varphi$ is of real type, as in the case of the defocusing NLS equation, the periodic spectrum is real, the eigenvalues are characterized by Floquet theory, and the lexicographical ordering reduces to the ordering of real numbers

$$
\cdots \leqslant \lambda_{n-1}^{+}<\lambda_{n}^{-} \leqslant \lambda_{n}^{\bullet} \leqslant \lambda_{n}^{+}<\lambda_{n+1}^{-} \leqslant \cdots
$$

Each $\varphi \in L_{r}^{2}$ has an open neighborhood $V_{\varphi}$ within $L_{c}^{2}$ for which there exist disjoint closed disks $\left(U_{n}\right)_{n \in \mathbb{Z}}$ centered on the real axis with the properties:

(i) $\lambda_{n}^{ \pm}(\psi)$ and $\lambda_{n}^{\bullet}(\psi)$ are contained in the interior of $U_{n}$ for every $\psi \in V_{\varphi}$,

(ii) there exists a constant $c \geqslant 1$ such that for $m \neq n$,

$$
c^{-1}|m-n| \leqslant \operatorname{dist}\left(U_{n}, U_{m}\right) \leqslant c|m-n|,
$$

(iii) $U_{n}=\{|\lambda-n \pi| \leqslant \pi / 4\}$ for $|n|$ sufficiently large.

Such disks are called isolating neighborhoods. The union of all $V_{\varphi}$ defines an open and connected neighborhood of $L_{r}^{2}$ within $L_{c}^{2}$ and is denoted by $W$. Throughout this text, $V_{\varphi}$ denotes a neighborhood of $\varphi$ such that a common set of isolating neighborhoods for all $\psi \in V_{\varphi}$ exists. 
Following Flaschka and McLaughlin's [8] approach for the KdV equation, one can define action variables for the defocusing NLS equation by Arnold's formula-see also [23]

$$
I_{n}=\frac{1}{\pi} \int_{a_{n}} \frac{\lambda \Delta^{\bullet}(\lambda)}{\sqrt{\Delta^{2}(\lambda)-4}} \mathrm{~d} \lambda .
$$

Here $a_{n}$ denotes a cycle around $\left(\lambda_{n}^{-}, \lambda_{n}^{+}\right)$on the spectral curve

$$
C_{\varphi}=\left\{(\lambda, z): z^{2}=\Delta^{2}(\lambda, \varphi)-4\right\} \subset \mathbb{C},
$$

on which the square root $\sqrt{\Delta^{2}(\lambda)-4}$ is defined. This curve is another spectral invariant associated with $\varphi$, and an open Riemann surface of infinite genus if and only if the periodic spectrum of $\varphi$ is simple. To avoid the technicalities involved with this curve, we follow the approach presented in [10] and fix proper branches of the square root which allows us to reduce the definition of the actions to standard contour integrals in the complex plane.

First, we denote by $\sqrt[+]{ }$ the principal branch of the square root on the complex plane minus the ray $(-\infty, 0]$. Second, we define the standard root

$$
\varsigma_{n}(\lambda)=\sqrt[s]{\left(\lambda_{n}^{+}-\lambda\right)\left(\lambda_{n}^{-}-\lambda\right)}, \quad \lambda \notin\left[\lambda_{n}^{-}, \lambda_{n}^{+}\right]
$$

by the condition

$$
\varsigma_{n}(\lambda):=\left(\tau_{n}-\lambda\right) \sqrt[+]{1-\gamma_{n}^{2} / 4\left(\tau_{n}-\lambda\right)}, \quad \tau_{n}=\left(\lambda_{n}^{-}+\lambda_{n}^{+}\right) / 2
$$

for all $|\lambda|$ sufficiently large. For any $\varphi \in W$, the standard root is analytic in $\lambda$ on $\mathbb{C} \backslash\left[\lambda_{n}^{-}, \lambda_{n}^{+}\right]$ and in $(\lambda, \psi)$ on $\left(\mathbb{C} \backslash U_{n}\right) \times V_{\varphi}$. Thirdly, we define the canonical root $\sqrt[c]{\Delta^{2}(\lambda)-4}$ by the product representation

$$
\sqrt[c]{\Delta^{2}(\lambda)-4}:=2 \mathrm{i} \prod_{m \in \mathbb{Z}} \frac{\varsigma_{m}(\lambda)}{\pi_{m}} .
$$

For any $\varphi \in W$, this root is analytic in $\lambda$ on $\mathbb{C} \backslash \bigcup_{\gamma_{n} \neq 0}\left[\lambda_{n}^{-}, \lambda_{n}^{+}\right]$and in $(\lambda, \psi)$ on $(\mathbb{C} \backslash$ $\left.\bigcup_{n \in \mathbb{Z}} U_{n}\right) \times V_{\varphi}$-see [10, Section 12] for all the details.

The $n$th NLS action variable of $\varphi \in W$ is then given by

$$
I_{n}:=\frac{1}{\pi} \int_{\Gamma_{n}} \frac{\lambda \Delta^{\bullet}(\lambda)}{\sqrt[c]{\Delta^{2}(\lambda)-4}} \mathrm{~d} \lambda,
$$


where $\Gamma_{n}$ denotes any sufficiently close circuit around $\left[\lambda_{n}^{-}, \lambda_{n}^{+}\right]$. More generally, the $n$th action on level $k \geqslant 1$ is given by

$$
J_{n, k}:=\frac{1}{k \pi} \int_{\Gamma_{n}} \frac{\lambda^{k} \Delta^{\bullet}(\lambda)}{\sqrt[c]{\Delta^{2}(\lambda)-4}} \mathrm{~d} \lambda .
$$

It was shown in [10] and, for convenience of the reader, will be reproved in the sequel that each action variable is analytic on $W$ and vanishes if and only if $\gamma_{n}$ is zero.

If $\varphi$ is of real type, then all actions are real and those on odd levels, such as $J_{n, 1}=I_{n}$, are nonnegative. Moreover, the actions on level one are well known to satisfy the trace formula,

$$
\sum_{n \in \mathbb{Z}} I_{n}(\varphi)=\mathcal{H}_{1}(\varphi)=\frac{1}{2}\|\varphi\|_{0}^{2}=\frac{1}{2} \int_{\mathbb{T}}\left(\left|\varphi_{-}\right|^{2}+\left|\varphi_{+}\right|^{2}\right) \mathrm{d} x
$$

Similar trace formulae have been derived by McKean and Vaninsky [23] expressing the actions on any level $k \geqslant 1$ in terms of Hamiltonians of the NLS hierarchy. The first three Hamiltonians of this hierarchy are

$$
\begin{aligned}
& \mathcal{H}_{1}(\varphi)=\int_{\mathbb{T}} \varphi_{-} \varphi_{+} \mathrm{d} x, \\
& \mathcal{H}_{2}(\varphi)=\frac{1}{2} \int_{\mathbb{T}}\left(\varphi_{-}^{\prime} \varphi_{+}-\varphi_{-} \varphi_{+}^{\prime}\right) \mathrm{d} x, \\
& \mathcal{H}_{3}(\varphi)=\int_{\mathbb{T}}\left(\varphi_{-}^{\prime} \varphi_{+}^{\prime}+\varphi_{+}^{2} \varphi_{-}^{2}\right) \mathrm{d} x,
\end{aligned}
$$

and in general, for any sufficiently regular $\varphi \in L_{c^{\prime}}^{2}$

$$
\mathcal{H}_{k+1}(\varphi)=\int_{\mathbb{T}}\left(-\varphi_{-} \varphi_{+}^{(k)}+q_{k}\left(\varphi, \ldots, \varphi^{(k-1)}\right)\right) \mathrm{d} x, \quad k \geqslant 1,
$$

with $q_{k}$ being a canonically determined polynomial in $\varphi$ and its first $k-1$ derivativessee Appendix 2. The following version of the trace formula is taken from [10].

Theorem 2.1 (Trace formula). For any $k \geqslant 2$ and any $\varphi \in H_{c}^{k-1} \cap W$,

$$
\sum_{n \in \mathbb{Z}} J_{n, k}(\varphi)=-\frac{1}{(2 \mathrm{i})^{k-1}} \mathcal{H}_{k}(\varphi) .
$$

In particular, for every sufficiently regular real-type potential

$$
\sum_{n \in \mathbb{Z}} J_{n, 2 m+1}(\varphi)=\frac{1}{4^{m}} \int_{\mathbb{T}}\left(\left|\varphi^{(m)}\right|^{2}+\cdots\right) \mathrm{d} x, \quad m \geqslant 0 .
$$


This identity is used in Sections 4 and 5 to estimate the actions on level $2 m+1$ in terms of $\|\varphi\|_{m}$. In order to obtain thereof estimates for the action variables on level 1, a detailed analysis of the analytical properties of the action integrand is necessary. To this end, we define for any $\varphi \in W$ on $\left(\mathbb{C} \backslash \bigcup_{n \in \mathbb{Z}} U_{n}\right) \times V_{\varphi}$ the complex 1-form

$$
\omega(\lambda, \psi):=\frac{\Delta^{\bullet}(\lambda, \psi)}{\sqrt[c]{\Delta^{2}(\lambda, \psi)-4}} \mathrm{~d} \lambda=-\mathrm{i} \prod_{m \in \mathbb{Z}} \frac{\lambda_{m}^{\bullet}(\psi)-\lambda}{\varsigma_{m}(\lambda, \psi)} \mathrm{d} \lambda .
$$

We call a path in the complex plane admissible for $\varphi \in L_{c}^{2}$ if, except possibly at its endpoints, it does not intersect any gap $\left[\lambda_{n}^{-}(\varphi), \lambda_{n}^{+}(\varphi)\right]$.

Lemma 2.2. For each $\varphi \in W$, the 1 -form $\omega$ has the following properties:

(i) $\omega$ is analytic on $\left(\mathbb{C} \backslash \bigcup_{n \in \mathbb{Z}} U_{n}\right) \times V_{\varphi}$,

(ii) $\omega(\cdot, \varphi)$ is analytic on $\mathbb{C} \backslash \bigcup_{\gamma_{n} \neq 0}\left[\lambda_{n}^{-}, \lambda_{n}^{+}\right]$, and

(iii) for any admissible path from $\lambda_{n}^{-}$to $\lambda_{n}^{+}$in $U_{n}$,

$$
\int_{\lambda_{n}^{-}}^{\lambda_{n}^{+}} \omega=0
$$

In particular, for any closed circuit $\Gamma_{n}$ in $U_{n}$ around $\left[\lambda_{n}^{-}, \lambda_{n}^{+}\right]$,

$$
\int_{\Gamma_{n}} \omega=0
$$

Proof. Since $\Delta^{2}(\lambda, \psi)-4$ vanishes if and only if $\lambda$ is a periodic eigenvalue, and numerator and dominator of $\omega(\lambda, \psi)$ are analytic on $\left(\mathbb{C} \backslash \bigcup_{n \in \mathbb{Z}} U_{n}\right) \times V_{\varphi}$, the first claim follows immediately.

By the same reasoning, $\omega(\cdot, \varphi)$ is analytic on $\mathbb{C} \backslash \bigcup_{n \in \mathbb{Z}}\left[\lambda_{n}^{-}, \lambda_{n}^{+}\right]$. Moreover, if the $n$th gap is collapsed, that is, $\lambda_{n}^{+}=\lambda_{n}^{-}=: \lambda_{n}$, then $\Delta^{2}(\lambda)-4$ has a double root at $\lambda_{n}$, and hence

$$
0=\Delta\left(\lambda_{n}\right) \Delta^{\bullet}\left(\lambda_{n}\right)=2(-1)^{n} \Delta^{\bullet}\left(\lambda_{n}\right) .
$$

As $\Delta^{\bullet}(\lambda)$ has a single root in $U_{n}$, namely $\lambda_{n^{\prime}}^{\bullet}$, we conclude $\lambda_{n}^{\bullet}=\lambda_{n}=\lambda_{n}^{ \pm}$, and the $n$th term in the product representations of $\Delta^{\bullet}$ and $\sqrt[c]{\Delta^{2}-4}$ cancels. So $\omega(\cdot, \varphi)$ is analytic on all of $U_{n}$, and the second claim follows.

We first consider the case where $\varphi$ is of real type. Then $(-1)^{n} \Delta(\lambda, \varphi) \geqslant 2$ on $\left[\lambda_{n}^{-}, \lambda_{n}^{+}\right]$, as shown in [10], and hence

$$
\min _{\lambda_{n}^{-} \leqslant \lambda \leqslant \lambda_{n}^{+}}(-1)^{n} \Delta(\lambda, \varphi)-\sqrt[+]{\Delta^{2}(\lambda, \varphi)-4}>0
$$


Thus, if we choose a circuit $\Gamma$ sufficiently close to $\left[\lambda_{n}^{-}, \lambda_{n}^{+}\right]$, and a sufficiently small neighborhood $V \subset V_{\varphi}$ of $\varphi$, then $\left[\lambda_{n}^{-}(\psi), \lambda_{n}^{+}(\psi)\right]$ is enclosed by $\Gamma$ and the real part of $(-1)^{n}\left(\Delta(\lambda, \psi)-\sqrt[c]{\Delta^{2}(\lambda, \psi)-4}\right)$ is positive on that circuit for all $\psi \in V$. In consequence, the principal branch of the logarithm

$$
l_{n}(\lambda, \psi)=\log \frac{(-1)^{n}}{2}\left(\Delta(\lambda, \psi)+\sqrt[c]{\Delta^{2}(\lambda, \psi)-4}\right)
$$

is analytic in a neighborhood of $\Gamma$ and $\mathrm{d} l_{n}=\omega$. It follows that the analytic functional $\psi \mapsto \int_{\partial U_{n}} \omega$ vanishes on an open neighborhood of $\varphi$, and hence on all of $V_{\varphi}$. Since $W=$ $\bigcup_{\varphi \in L_{r}^{2}} V_{\varphi}$, it follows that $\int_{\Gamma_{n}} \omega=0$ for every $\varphi \in W$.

Finally, consider $\int_{\lambda_{n}^{-}}^{\lambda_{n}^{+}} \omega$ with the path of integration chosen to be admissible. As $\omega$ is closed around the gap, the integral does not depend on the chosen admissible path. Suppose $\gamma_{n} \neq 0$, then by the product representation (4) of $\omega$,

$$
\int_{\lambda_{n}^{-}}^{\lambda_{n}^{+}} \omega=-\mathrm{i} \int_{\lambda_{n}^{-}}^{\lambda_{n}^{+}} \frac{\lambda_{n}^{\bullet}-\lambda}{\varsigma_{n}(\lambda)} \chi_{n}(\lambda) \mathrm{d} \lambda, \quad \chi_{n}(\lambda):=\prod_{m \neq n} \frac{\lambda_{m}^{\bullet}-\lambda}{\zeta_{m}(\lambda)},
$$

where $\chi_{n}(\lambda)$ is analytic on $\mathbb{C} \backslash \bigcup_{m \neq n}\left[\lambda_{m}^{-}, \lambda_{m}^{+}\right]$-see [10, Section 12]. Further, by the definition of the standard root (1), the function

$$
z \mapsto \varsigma_{n}\left(\tau_{n}+z \gamma_{n} / 2\right)=-z \sqrt[+]{1-z^{-2}} \gamma_{n} / 2
$$

is analytic on $\mathbb{C} \backslash[-1,1]$. For $s \in(-1,1)$, consider $z_{S}=s \pm \mathrm{i} \varepsilon$ to conclude

$$
\varsigma_{n}\left(\tau_{n}+(s \pm \mathrm{i} 0) \gamma_{n} / 2\right)=\mp \mathrm{i} \sqrt[+]{1-s^{2}} \gamma_{n} / 2
$$

In particular, if $\alpha_{1}$ and $\alpha_{2}$ are admissible paths from $\lambda_{n}^{-}$to $\lambda_{n}^{+}$running on different sides of $\left[\lambda_{n}^{-}, \lambda_{n}^{+}\right]$, then the integrand takes the opposite sign on these paths and hence

$$
\int_{\alpha_{1}} \omega=-\int_{\alpha_{2}} \omega
$$

On the other hand, as $\int_{\partial U_{n}} \omega=0$, the integral is independent of the chosen path $\alpha_{i}$ and thus needs to be zero.

If we write the action variables as

$$
J_{n, k}=\frac{1}{k \pi} \int_{\Gamma_{n}} \lambda^{k} \omega
$$


then the analyticity on $W$ is evident. To proceed, we need to find a globally defined primitive of $\omega$. So for $\varphi \in W$, we define on $\left(\mathbb{C} \backslash \bigcup_{n \in \mathbb{Z}} U_{n}\right) \times V_{\varphi}$ the mapping

$$
F(\lambda, \psi):=\frac{1}{2}\left(\int_{\lambda_{0}^{-}(\psi)}^{\lambda} \omega(\mu, \psi)+\int_{\lambda_{0}^{+}(\psi)}^{\lambda} \omega(\mu, \psi)\right),
$$

where the paths of integration are chosen to be admissible. These improper integrals exist, as for $\gamma_{0}=0$ the integrand is analytic on $U_{0}$, while for $\gamma_{0} \neq 0$ it is of the form $1 / \sqrt{\lambda_{0}^{ \pm}-\lambda}$ locally around $\lambda_{0}^{ \pm}$. By Lemma 2.2, they are also independent of the chosen admissible path. Hence, $F$ is well defined and one has

$$
F(\lambda, \psi)=\int_{\lambda_{0}^{-}(\psi)}^{\lambda} \omega(\mu, \psi)=\int_{\lambda_{0}^{+}(\psi)}^{\lambda} \omega(\mu, \psi) .
$$

Even though the eigenvalues $\lambda_{0}^{ \pm}$are, due to their lexicographical ordering, not even continuous on $W$, the mapping $F$ turns out to be differentiable.

Lemma 2.3. For every $\varphi \in W$, we have that

(i) $\quad F$ is analytic on $\left(\mathbb{C} \backslash \bigcup_{n \in \mathbb{Z}} U_{n}\right) \times V_{\varphi}$ and

(ii) $F(\cdot, \varphi)$ can be continuously extended onto $\mathbb{C} \backslash \bigcup_{\gamma_{n} \neq 0}\left(\lambda_{n}^{-}, \lambda_{n}^{+}\right)$with

$$
F\left(\lambda_{n}^{+}, \varphi\right)=F\left(\lambda_{n}^{-}, \varphi\right), \quad n \in \mathbb{Z}
$$

(iii) If, in addition, $\varphi \in L_{r}^{2}$, then locally around $\left[\lambda_{n}^{-}, \lambda_{n}^{+}\right]$

$$
F(\lambda)=l_{n}(\lambda)-\operatorname{in} \pi, \quad l_{n}(\lambda)=\log \frac{(-1)^{n}}{2}\left(\Delta(\lambda)+\sqrt[c]{\Delta^{2}(\lambda)-4}\right) .
$$

In particular, for any real $\lambda_{n}^{-}<\lambda<\lambda_{n}^{+}$,

$$
F(\lambda \pm \mathrm{i} 0)= \pm f_{n}(\lambda)-\mathrm{i} n \pi, \quad f_{n}(\lambda)=\cosh ^{-1}\left((-1)^{n} \Delta(\lambda) / 2\right)
$$

Clearly, $f_{n}$ is continuous on $\left[\lambda_{n}^{-}, \lambda_{n}^{+}\right]$, strictly positive on $\left(\lambda_{n}^{-}, \lambda_{n}^{+}\right)$, and vanishes at the boundary points.

(iv) At the zero potential, one has $F(\lambda, 0)=-i \lambda$.

Proof. The proof of (i) is standard but a bit technical and can be found in Appendix 1, and claim (ii) follows immediately from the properties of $\omega$.

(iii) Note that locally around $\left[\lambda_{n}^{-}, \lambda_{n}^{+}\right]$both $l_{n}$ and $F$ are primitives of $\omega$ and hence are identical up to a additive constant which may depend on $\varphi$. Clearly, $l_{n}\left(\lambda_{n}^{ \pm}\right)=0$. On the 
other hand, since $\int_{\lambda_{k}^{-}}^{\lambda_{+}^{+}} \omega=0$ for any $k$,

$$
F\left(\lambda_{n}^{ \pm}\right)=\sum_{k=0}^{n-1} \int_{\lambda_{k}^{+}}^{\lambda_{k+1}^{-}} \omega, \quad F\left(\lambda_{-n}^{ \pm}\right)=\sum_{k=0}^{n-1} \int_{\lambda_{-k}^{-}}^{\lambda_{-k-1}^{+}} \omega, \quad n>0 .
$$

As $\mathrm{i}(-1) \sqrt[k]{\Delta^{2}(\lambda)-4}>0$ for $\lambda_{k}^{+}<\lambda<\lambda_{k+1}^{-}$- see [10, Section 12] -we find

$$
\int_{\lambda_{k}^{+}}^{\lambda_{k+1}^{-}} \omega=\mathrm{i}(-1)^{k} \int_{\lambda_{k}^{+}}^{\lambda_{k+1}^{-}} \frac{\dot{\Delta}(\lambda)}{\sqrt[+]{4-\Delta^{2}(\lambda)}} \mathrm{d} \lambda=\left.\mathrm{i}(-1)^{k} \arcsin \frac{\Delta(\lambda)}{2}\right|_{\lambda_{k}^{+}} ^{\lambda_{k+1}^{-}}=-\mathrm{i} \pi .
$$

Consequently, $F\left(\lambda_{n}^{ \pm}\right)=-\mathrm{i} n \pi$ and $F-l_{n} \equiv-\mathrm{i} n \pi$. Finally, $\pm(-1) \sqrt[n]{\Delta^{2}(\lambda \pm \mathrm{i} 0)-4}>0$ for $\lambda_{n}^{-}<\lambda<\lambda_{n}^{+}$hence

$$
F(\lambda \pm \mathrm{i} 0)=\log \frac{1}{2}\left((-1)^{n} \Delta(\lambda) \pm \sqrt[+]{\Delta^{2}(\lambda)-4}\right)-\mathrm{i} n \pi= \pm f_{n}(\Delta(\lambda) / 2)-\mathrm{i} n \pi .
$$

(iv) At the zero potential, $\omega(\lambda, 0)=-\mathrm{i} d \lambda$ which is evident from the product representation (4) of $\omega$.

Given $\varphi \in W$, we can integrate by parts in (6) to obtain

$$
J_{n, k}=\frac{1}{k \pi} \int_{\Gamma_{n}} \lambda^{k} \omega=-\frac{1}{\pi} \int_{\Gamma_{n}} \lambda^{k-1} F(\lambda) \mathrm{d} \lambda .
$$

Clearly, $J_{n, k}$ vanishes if $\gamma_{n}=0$ in view of the analyticity of the integrand. Further, provided $\varphi$ is of real type, then we may shrink the contour of integration to $\left[\lambda_{n}^{-}, \lambda_{n}^{+}\right]$, and use the properties of $F$ to the effect that

$$
J_{n, k}=\frac{2}{\pi} \int_{\lambda_{n}^{-}}^{\lambda_{n}^{+}} \lambda^{k-1} f_{n}(\lambda) \mathrm{d} \lambda
$$

Thus, on $L_{r}^{2}$ all actions are real and those on odd levels are nonnegative. Moreover, by the mean value theorem,

$$
J_{n, 2 m+1}=\zeta_{n, m}^{2 m} I_{n}
$$

for some $\zeta_{n, m} \in\left[\lambda_{n}^{-}, \lambda_{n}^{+}\right]$. Recall that $\lambda_{n}^{ \pm} \sim n \pi$ hence for any $m \geqslant 0$, we have $\zeta_{n, m}^{2 m} \sim(n \pi)^{2 m}$ asymptotically as $|n| \rightarrow \infty$. A quantitative estimate of the high-level actions $J_{n, 2 m+1}$ in terms of the actions $I_{n}$ will be obtained in the next section.

Finally, consider the case of a potential with only finitely many open gaps. Then $F$ is analytic outside some sufficiently large circle and thus admits a Laurent expansion around zero. The coefficients of this expansion turn out to be the Hamiltonians of the NLS hierarchy. 
Lemma 2.4. For any finite gap potential $\varphi \in W$, there exists $\Lambda>0$ such that

$$
F(\lambda, \varphi)=-\mathrm{i} \lambda-\frac{\mathcal{H}_{1}(\varphi)}{2 \mathrm{i} \lambda}+\sum_{n \geqslant 2} \frac{\mathcal{H}_{n}(\varphi)}{(2 \mathrm{i} \lambda)^{n}}, \quad|\lambda|>\Lambda
$$

Proof. We attribute the claim to the asymptotic expansion of $\cosh ^{-1}(\Delta / 2)$ along the real axis-see [10, 17]. By the basic estimates for the discriminant

$$
\Delta(\mathrm{i} \tau, \varphi)=2 \cosh \tau+o\left(\mathrm{e}^{\tau}\right), \quad \tau \rightarrow \infty
$$

Hence, on an open neighborhood $U$ of $\mathrm{i}\left[\tau_{0}, \infty\right)$ for $\tau_{0}>0$ sufficiently large $\cosh ^{-1}$ $(\Delta(\lambda, \varphi) / 2)$ is well defined. Here $\cosh ^{-1}$ denotes the principal branch of the inverse of cosh which is defined on $\mathbb{C} \backslash(-\infty, 1)$ and real valued on $[1, \infty)$. On this neighborhood $U$, the $\lambda$-derivatives of $\cosh ^{-1}(\Delta / 2)$ and $F$ coincide except for possibly the sign of

$$
\Re \sqrt[c]{\Delta^{2}(\mathrm{i} \tau)-4}, \quad \tau \geqslant \tau_{0} .
$$

This sign is locally constant in $\varphi$ provided $\tau \geqslant \tau_{0}$ and, as the straight line $\{t \varphi: 0 \leqslant t \leqslant 1\}$ is compact in $L_{c}^{2}$, it can be determined by deforming $\varphi$ to the zero potential. With

$$
\sqrt[c]{\Delta^{2}(\mathrm{i} \tau, 0)-4}=2 \mathrm{i} \prod_{m \in \mathbb{Z}} \frac{\varsigma_{m}(\mathrm{i} \tau, 0)}{\pi_{m}}=2 \mathrm{i} \prod_{m \in \mathbb{Z}} \frac{m \pi-\mathrm{i} \tau}{\pi_{m}}=2 \sinh \tau,
$$

we conclude the sign is positive on $U$, and consequently

$$
\cosh ^{-1} \frac{\Delta(\lambda, \varphi)}{2}=F(\lambda, \varphi)+C(\varphi), \quad \lambda \in U,
$$

with an analytic function $c: W \rightarrow \mathbb{C}$. For $\varphi \in W$ fixed, the right-hand side is analytic on $\mathbb{C} \backslash \bigcup_{\gamma_{n} \neq 0}\left[\lambda_{n}^{-}, \lambda_{n}^{+}\right]$and continuous on $\mathbb{C} \backslash \bigcup_{\gamma_{n} \neq 0}\left(\lambda_{n}^{-}, \lambda_{n}^{+}\right)$, hence the left-hand side extends uniquely from $U$ onto the same domain. Moreover, $F$ vanishes at $\lambda_{0}^{ \pm}$, while

$$
\cosh ^{-1} \frac{\Delta\left(\lambda_{0}^{ \pm}, \varphi\right)}{2} \in\{\mathrm{i} m \pi: m \in \mathbb{Z}\} .
$$

Thus, $c(\varphi)=\mathrm{i} m \pi$, and since $c$ is continuous, this $m$ is fixed on all of $W$. Evaluating at the zero potential reveals $\cosh ^{-1}(\Delta(\lambda, 0) / 2)=-\mathrm{i} \lambda=F(\lambda, 0)$ hence $c \equiv 0$.

If $\varphi$ is a finite gap potential, then $\cosh ^{-1}(\Delta / 2)=F$ is analytic outside a sufficiently large circle enclosing all open gaps, and has the following asymptotic expansion along the real axis [17]

$$
\cosh ^{-1} \frac{\Delta(\lambda, \varphi)}{2}=-\mathrm{i} \lambda-\frac{\mathcal{H}_{1}(\varphi)}{2 \mathrm{i} \lambda}+\sum_{n \geqslant 2} \frac{\mathcal{H}_{n}(\varphi)}{(2 \mathrm{i} \lambda)^{n}}, \quad \lambda \rightarrow \pm \infty .
$$

This proves the claim. 


\section{Localizing the Zakharov-Shabat Spectrum}

The goal for this section is to provide a sufficiently accurate localization of the spectrum of the Zakharov-Shabat operator

$$
L(\varphi)=\left(\begin{array}{cc}
\mathrm{i} & \\
& -\mathrm{i}
\end{array}\right) \frac{\mathrm{d}}{\mathrm{d} x}+\left(\begin{array}{ll} 
& \varphi_{-} \\
\varphi_{+} &
\end{array}\right),
$$

allowing to quantify the asymptotic relation $J_{n, 2 m+1} \sim(n \pi)^{2 m} I_{n}$. Since one can translate the spectrum of $\varphi$ without changing the $L^{2}$-norm, one cannot obtain a uniform localization on bounded subsets of $L_{c}^{2}$. Instead, we have to impose some regularity on $\varphi$.

Theorem 3.1. Suppose $\varphi \in H_{c}^{1}$, then for each $\langle n\rangle \geqslant 8\|\varphi\|_{1}^{2}$,

$$
\left|\lambda_{n}^{ \pm}-n \pi\right| \leqslant \frac{\|\varphi\|_{1}^{2}}{\langle n\rangle}+\frac{\sqrt{2}\|\varphi\|_{1}}{\langle 2 n\rangle} \leqslant \pi / 5
$$

while the remaining eigenvalues are contained in the box

$$
\left\{\lambda \in \mathbb{C}:|\mathfrak{R} \lambda| \leqslant\left(8\|\varphi\|_{1}^{2}-1 / 2\right) \pi,|\Im \lambda| \leqslant\|\varphi\|_{1}\right\} .
$$

Remark. In [22], Li and McLaughlin obtained a localization for $\varphi$ in $H_{c}^{1}$ where the bound on the threshold of $\langle n\rangle$ is exponential in the norm of $\varphi$. With a focus on lowering the regularity assumptions on $\varphi$ rather than improving the threshold for $\varphi$ smooth, this result was gradually improved by several authors-see, for example, Mityagin [24] and the references therein. The novelty of Theorem 3.1 consists in providing a threshold for $\langle n\rangle$ which is quadratic in the norm of $\varphi$.

The proof is based on a Lyapunov-Schmidt decomposition introduced by Kappeler and Mityagin [12]—see also [9, 11]: For the zero potential each $n \pi, n \in \mathbb{Z}$, is a double eigenvalue of $L$ with eigenfunctions $e_{n}^{+}:=\left(0, \mathrm{e}^{\mathrm{i} n \pi x}\right)$ and $e_{n}^{-}:=\left(\mathrm{e}^{-\mathrm{i} n \pi x}, 0\right)$. Thus, for a nonzero potential, provided $|n|$ is sufficiently large, we expect exactly two eigenvalues which are close to $n \pi$ and whose eigenfunctions are close to the linear span of $e_{n}^{+}$and $e_{n}^{-}$. This suggest to separate these modes from the others by a Lyapunov-Schmidt reduction.

To set the stage, we cover the complex plane with the closed strips

$$
\mathfrak{U}_{n}:=\{\lambda:|\mathfrak{R} \lambda-n \pi| \leqslant \pi / 2\},
$$

and consider the Hilbert space of complex 2-periodic $L^{2}$-functions

$$
L_{*}^{2}=\mathcal{P}_{n} \oplus \mathcal{Q}_{n}=\operatorname{sp}\left\{e_{n}^{+}, e_{n}^{-}\right\} \oplus \overline{\operatorname{sp}}\left\{e_{k}^{+}, e_{k}^{-}: k \neq n\right\} .
$$

The orthogonal projections onto $\mathcal{P}_{n}$ and $\mathcal{Q}_{n}$ are denoted by $P_{n}$ and $Q_{n}$, respectively. 
To proceed, write the eigenvalue equation $L f=\lambda f$ in the form

$$
A_{\lambda} f=\Phi f
$$

with the unbounded operators

$$
A_{\lambda}:=\lambda-\left(\begin{array}{ll}
\mathrm{i} & \\
& -\mathrm{i}
\end{array}\right) \frac{\mathrm{d}}{\mathrm{d} x}, \quad \Phi:=\left(\begin{array}{ll} 
& \varphi_{-} \\
\varphi_{+} &
\end{array}\right) .
$$

Since $A_{\lambda}$ leaves the spaces $\mathcal{P}_{n}$ and $\mathcal{Q}_{n}$ invariant, by writing

$$
f=u+v=P_{n} f+Q_{n} f
$$

we can decompose the equation $A_{\lambda} f=\Phi f$ into the two equations

$$
A_{\lambda} u=P_{n} \Phi(u+v), \quad A_{\lambda} v=Q_{n} \Phi(u+v)
$$

called the $P$ - and the $Q$-equation, respectively.

We first consider the $Q$-equation on $\mathfrak{U}_{n}$. One checks that for $m \neq n$

$$
\min _{\lambda \in \mathfrak{U}_{n}}|\lambda-m \pi| \geqslant|n-m| \geqslant 1
$$

hence it follows with $A_{\lambda} e_{m}^{ \pm}=(\lambda-m \pi) e_{m}^{ \pm}$that the restriction of $A_{\lambda}$ to $\mathcal{Q}_{n}$ is boundedly invertible for all $\lambda \in \mathfrak{U}_{n}$. Therefore, multiplying the $Q$-equation from the left by $\Phi A_{\lambda}^{-1}$, gives

$$
\Phi v=T_{n} \Phi(u+v)
$$

with $T_{n}:=\Phi A_{\lambda}^{-1} Q_{n}$. The latter identity may be written as

$$
\left(I-T_{n}\right) \Phi v=T_{n} \Phi u
$$

hence solving the $Q$ equation amounts to inverting ( $\left.I-T_{n}\right)$.

We consider operator norms induced by shifted weighted norms $[13,26]$. Let $H_{i}^{w}$ denote the space of complex 2-periodic functions $u=\sum_{m \in \mathbb{Z}} u_{m} e_{m}$ equipped with the $i$-shifted $H^{w}$-norm given by

$$
\|u\|_{w ; i}^{2}:=\left\|u e_{i}\right\|_{w}^{2}=\sum_{m \in \mathbb{Z}} w_{m+i}^{2}\left|u_{m}\right|^{2}, \quad e_{m}(x):=\mathrm{e}^{\mathrm{i} m \pi x} .
$$

On the space $H_{i, c}^{w}:=H_{i}^{w} \times H_{i}^{w}$ of 2-periodic functions with values in $\mathbb{C}^{2}$,

$$
f=\left(f_{-}, f_{+}\right)=\sum_{n \in \mathbb{Z}}\left(f_{n}^{-} e_{n}^{-}+f_{n}^{+} e_{n}^{+}\right)=\sum_{n \in \mathbb{Z}}\left(f_{n}^{-} e_{-n}^{-}, f_{n}^{+} e_{n}\right),
$$


the $i$-shifted norm is defined by

$$
\|f\|_{w ; i}^{2}:=\left\|f_{-}\right\|_{w ;-i}^{2}+\left\|f_{+}\right\|_{w ; i}^{2}=\sum_{m \in \mathbb{Z}} w_{m+i}^{2}\left(\left|f_{m}^{-}\right|^{2}+\left|f_{m}^{+}\right|^{2}\right) .
$$

Lemma 3.2. If $\varphi \in H_{c}^{w}$ with $w \in \mathcal{M}$, then for any $n, i \in \mathbb{Z}$ and any $\lambda \in \mathfrak{U}_{n}$

$$
T_{n}=\Phi A_{\lambda}^{-1} Q_{n}: H_{i, c}^{w} \rightarrow H_{-i, c}^{w}
$$

is bounded and satisfies the estimate

$$
\left\|T_{n} f\right\|_{w ; i} \leqslant 2\|\varphi\|_{w}\|f\|_{w ;-i}
$$

Proof. Write $T_{n} f=\Phi g$ with $g=A_{\lambda}^{-1} Q_{n} f$. Since the restriction of $A_{\lambda}$ to $\mathcal{Q}_{n}$ is boundedly invertible, the function

$$
g=A_{\lambda}^{-1} Q_{n} f=\sum_{m \neq n}\left(\frac{f_{m}^{-}}{\lambda-m \pi} e_{m}^{-}+\frac{f_{m}^{+}}{\lambda-m \pi} e_{m}^{+}\right)=\left(g_{-}, g_{+}\right)
$$

is well defined. By Hlder's inequality, we obtain for the weighted $\ell^{1}$-norm

$$
\left\|g_{+} e_{-i}\right\|_{\ell_{w}^{1}}=\sum_{m \neq n} \frac{w_{m-i}\left|f_{m}^{+}\right|}{|\lambda-m \pi|} \leqslant\left(\sum_{m \neq n} \frac{1}{|n-m|^{2}}\right)^{1 / 2}\left\|f_{+}\right\|_{w ;-i}<2\left\|f_{+}\right\|_{w ;-i}
$$

uniformly for $\lambda \in \mathfrak{U}_{n} ;$ similarly $\left\|g_{-} e_{i}\right\|_{\ell_{w}^{1}} \leqslant 2\left\|f_{-}\right\|_{w ; i}$. Since

$$
\left\|T_{n} f\right\|_{w ; i}^{2}=\|\Phi g\|_{w ; i}^{2}=\left\|\varphi_{-} g_{+} e_{-i}\right\|_{w}^{2}+\left\|\varphi_{+} g_{-} e_{i}\right\|_{w}^{2},
$$

we can use standard inequalities for the convolution of sequences to obtain

$$
\left\|T_{n} f\right\|_{w ; i}^{2} \leqslant\|\varphi\|_{w}^{2}\left(\left\|g_{+} e_{-i}\right\|_{\ell_{w}^{1}}^{2}+\left\|g_{-} e_{i}\right\|_{\ell_{w}^{1}}^{2}\right) \leqslant 4\|\varphi\|_{w}^{2}\|f\|_{w ;-i}^{2} .
$$

Note that $T_{n} f$ is estimated with a shifted $H^{w}$-norm where the sign of the shift is opposite to the sign of the shifted $H^{w}$-norm of $f$. This fact will be crucial in the following. In particular, $T_{n}^{2}$ is bounded on $H_{i, c}^{w}$ and it turns out that $\left\|T_{n}^{2}\right\|_{w ; n}=o(1)$ as $|n| \rightarrow \infty$. Using a Neumann series, we then obtain the bounded invertibility of $\left(I-T_{n}\right)$ for $|n|$ sufficiently large, which solves the $Q$-equation. To exploit the regularity assumption $\varphi \in H_{c}^{1}$ of Theorem 3.1, we restrict ourselves to the subclass $\mathcal{M}^{1}$ of weights which have at least a linearly growing factor-see also [7, 9] for weights with factors $\langle n\rangle^{\delta}, 0<\delta<\frac{1}{2}$. 
Lemma 3.3. If $\varphi \in H_{c}^{w}$ with $w \in \mathcal{M}^{1}$, then for any $n \in \mathbb{Z}$ and any $\lambda \in \mathfrak{U}_{n}$,

$$
\left\|T_{n}^{2}\right\|_{w ; n} \leqslant \frac{4}{\langle n\rangle}\|\varphi\|_{w}^{2} .
$$

Proof. As in the preceding lemma, write $T_{n}^{2} f=\Phi g$ with

$$
g:=A_{\lambda}^{-1} Q_{n} \Phi A_{\lambda}^{-1} Q_{n} f
$$

A straightforward computation yields

$$
g=\sum_{k, l \neq n}\left(\frac{\varphi_{k+l}^{-}}{\lambda-k \pi} \frac{f_{l}^{+}}{\lambda-l \pi} e_{k}^{-}+\frac{\varphi_{k+l}^{+}}{\lambda-k \pi} \frac{f_{l}^{-}}{\lambda-l \pi} e_{k}^{+}\right)=\left(g_{-}, g_{+}\right),
$$

and our aim is to estimate the weighted $\ell^{1}$-norms $\left\|g_{+} e_{-n}\right\|_{\ell_{w}^{1}}$ and $\left\|g_{-} e_{n}\right\|_{\ell_{w}^{1}}$. By assumption $w=\langle n\rangle \cdot v$ with some submultiplicative weight $v$, hence

$$
w_{k-n} \leqslant \frac{\langle k-n\rangle}{\langle k+l\rangle\langle l+n\rangle} w_{k+l} w_{-l-n}, \quad k, l \in \mathbb{Z} .
$$

Consequently, for any $\lambda \in \mathfrak{U}_{n}$

$$
\left\|g_{+} e_{-n}\right\|_{\ell_{w}^{1}} \leqslant \sum_{k, l \neq n} \frac{\langle k-n\rangle}{\langle k+l\rangle|n-k|\langle l+n\rangle|n-l|} w_{k+l}\left|\varphi_{k+l}^{+}\right| w_{-l-n}\left|f_{l}^{-}\right|,
$$

and with Cauchy-Schwarz and Young's inequality for convolutions,

$$
\leqslant\left(\sum_{k, l \neq n} \frac{\langle k-n\rangle^{2}}{\langle k+l\rangle^{2}|n-k|^{2}\langle l+n\rangle^{2}|n-l|^{2}}\right)^{1 / 2}\|\varphi\|_{w}\left\|f_{-}\right\|_{w ;-n} .
$$

One further checks that

$$
\sum_{k \neq n} \frac{\langle k-n\rangle^{2}}{\langle k+l\rangle^{2}|n-k|^{2}} \leqslant 32 / 5, \quad \sum_{l \neq n} \frac{1}{\langle l+n\rangle^{2}|n-l|^{2}} \leqslant \frac{5 / 2}{\langle n\rangle^{2}} .
$$

Hence, we obtain for $\left\|g_{+} e_{-n}\right\|_{\ell_{w}^{1}}$ and similarly for $\left\|g_{-} e_{n}\right\|_{\ell_{w}^{1}}$,

$$
\left\|g_{+} e_{-n}\right\|_{\ell_{w}^{1}} \leqslant \frac{4}{\langle n\rangle}\|\varphi\|_{w}\left\|f_{-}\right\|_{w ;-n}, \quad\left\|g_{-} e_{n}\right\|_{\ell_{w}^{1}} \leqslant \frac{4}{\langle n\rangle}\|\varphi\|_{w}\left\|f_{+}\right\|_{w ; n} .
$$

Finally, with $\left\|T_{n}^{2} f\right\|_{w ; n}=\|\Phi g\|_{w ; n}$, this gives

$$
\left\|T_{n}^{2} f\right\|_{w ; n}^{2} \leqslant\|\varphi\|_{w}^{2}\left(\left\|g_{+} e_{-n}\right\|_{\ell_{1}^{w}}^{2}+\left\|g_{-} e_{n}\right\|_{\ell_{1}^{w}}^{2}\right) \leqslant \frac{16}{\langle n\rangle^{2}}\|\varphi\|_{w}^{4}\|f\|_{w ; n}^{2} .
$$


Consequently, $T_{n}^{2}$ is a $1 / 2$-contraction if $\langle n\rangle \geqslant 8\|\varphi\|_{w}^{2}$. In view of

$$
\hat{T}_{n}:=\left(I-T_{n}\right)^{-1}=\left(I+T_{n}\right)\left(I-T_{n}^{2}\right)^{-1},
$$

one then finds a unique solution

$$
\Phi v=\hat{T}_{n} T_{n} \Phi u
$$

of the $Q$-equation. In turn, as $I+\hat{T}_{n} T_{n}=\hat{T}_{n}$, the $P$-equation yields

$$
A_{\lambda} u=P_{n}\left(I+\hat{T}_{n} T_{n}\right) \Phi u=P_{n} \hat{T}_{n} \Phi u .
$$

Writing the latter as

$$
S_{n} u=0, \quad S_{n}: \mathcal{P}_{n} \rightarrow \mathcal{P}_{n}, \quad u \mapsto\left(A_{\lambda}-P_{n} \hat{T}_{n} \Phi\right) u,
$$

we immediately conclude that there exists the following relationship.

Lemma 3.4. For $\varphi \in H_{c}^{1}$ and $\langle n\rangle \geqslant 8\|\varphi\|_{1}^{2}$, a complex number $\lambda \in \mathfrak{U}_{n}$ is an eigenvalue of $L$ if and only if the determinant of $S_{n}$ vanishes.

Proof. Suppose $L f=\lambda f$, then by the preceding discussion $S_{n} u=0$. Conversely, define for any $u \in \mathcal{P}_{n}$,

$$
v=A_{\lambda}^{-1} Q_{n} \hat{T}_{n} \Phi u \in \mathcal{Q}_{n}
$$

Then $\Phi v=T_{n} \hat{T}_{n} \Phi u$ is well defined, and it follows with $\hat{T}_{n}=I+T_{n} \hat{T}_{n}$ that

$$
A_{\lambda} v=Q_{n} \hat{T}_{n} \Phi u=Q_{n} \Phi u+Q_{n} T_{n} \hat{T}_{n} \Phi u=Q_{n} \Phi(u+v)
$$

so the $Q$-equation is automatically satisfied. Moreover, if $S_{n} u=0$, then

$$
A_{\lambda} u=P_{n} \hat{T}_{n} \Phi u=P_{n}\left(I+T_{n} \hat{T}_{n}\right) \Phi u=P_{n} \Phi(u+v) .
$$

Hence also the $P$-equation is satisfied, and $\lambda$ is an eigenvalue of $L$ with eigenfunction $f=u+v$.

Recall that $P_{n}$ is the orthogonal projection onto the 2D space $\mathcal{P}_{n}$. The matrix representation of an operator $B$ on $\mathcal{P}_{n}$ is given by

$$
\left(\left\langle B e_{n}^{ \pm}, e_{n}^{ \pm}\right\rangle\right)_{ \pm, \pm}
$$


Therefore, we find for $S_{n}$ the representation

$$
A_{\lambda}=\left(\begin{array}{cc}
\lambda-n \pi & \\
& \lambda-n \pi
\end{array}\right), \quad P_{n} \hat{T}_{n} \Phi=\left(\begin{array}{cc}
a_{n}^{+} & b_{n}^{+} \\
b_{n}^{-} & a_{n}^{-}
\end{array}\right),
$$

with the coefficients of the latter matrix given by

$$
a_{n}^{ \pm}:=\left\langle\hat{T}_{n} \Phi e_{n}^{ \pm}, e_{n}^{ \pm}\right\rangle, \quad b_{n}^{ \pm}:=\left\langle\hat{T}_{n} \Phi e_{n}^{\mp}, e_{n}^{ \pm}\right\rangle
$$

We point out that these coefficients depend on $\lambda$ and $\varphi$. It has been observed in $[7,16]$ that these coefficients reflect certain symmetries of the Fourier coefficients of $\varphi$. We only need the fact that $a_{n}^{+}$and $a_{n}^{-}$coincide.

Lemma 3.5. Suppose $\varphi \in H_{c}^{1}$ and $\langle n\rangle \geqslant 8\|\varphi\|_{1}^{2}$, then for any $\lambda \in \mathfrak{U}_{n}$,

$$
a_{n}^{+}(\lambda)=a_{n}^{-}(\lambda) \equiv a_{n}(\lambda)
$$

Proof. Recall that $T_{n}=\Phi A_{\lambda}^{-1} Q_{n}$, and denote by $\bar{B} u:=\bar{B} \bar{u}$ the complex conjugation of operators. From evaluating the bounded diagonal operators $A_{\lambda}^{-1}$ and $Q_{n}$ at $e_{m}^{ \pm}$, and using the identity $\overline{e_{m}^{ \pm}}=P e_{m}^{\mp}$, we conclude

$$
\left(A_{\lambda}^{-1}\right)^{*}=P \overline{A_{\lambda}^{-1}} P=A_{\bar{\lambda}}^{-1}, \quad Q_{n}^{*}=P \bar{Q}_{n} P=Q_{n}, \quad P:=\left({ }_{1}{ }^{1}\right) .
$$

Since $A_{\lambda}^{-1}$ leaves $\mathcal{Q}_{n}$ invariant, and $P^{2}=I$, we find $\left(A_{\lambda}^{-1} Q_{n}\right)^{*}=P \overline{A_{\lambda}^{-1} Q_{n} P}$. With $\Phi^{*}=P \bar{\Phi} P$ this gives

$$
\left(T_{n} \Phi\right)^{*}=\Phi^{*}\left(A_{\lambda}^{-1} Q_{n}\right)^{*} \Phi^{*}=P \overline{T_{n} \Phi} P .
$$

Inspecting the Neumann expansion of $\hat{T}_{n} \Phi$ yields $\left(\hat{T}_{n} \Phi\right)^{*}=P \overline{\hat{T}_{n} \Phi} P$, thus

$$
a_{n}^{+}=\left\langle\hat{T}_{n} \Phi e_{n}^{+}, e_{n}^{+}\right\rangle=\left\langle e_{n}^{+},\left(\hat{T}_{n} \Phi\right)^{*} e_{n}^{+}\right\rangle=\left\langle P e_{n}^{+}, \overline{\hat{T}_{n} \Phi} P e_{n}^{+}\right\rangle=\left\langle\hat{T}_{n} \Phi e_{n}^{-}, e_{n}^{-}\right\rangle=a_{n}^{-}
$$

It follows that $S_{n}$ may be written as

$$
S_{n}(\lambda)=\left(\begin{array}{cc}
\lambda-n \pi-a_{n} & -b_{n}^{+} \\
-b_{n}^{-} & \lambda-n \pi-a_{n}
\end{array}\right) .
$$

As $T_{n}$ and $\Phi$ are anti-diagonal while $I$ and $T_{n}^{2}$ are diagonal, all even terms $\left\langle T_{n}^{2 k} \Phi e_{n}^{+}, e_{n}^{+}\right\rangle$ in the expansion of $a_{n}$ vanish. Using $\hat{T}_{n}=\left(I+T_{n}\right)\left(I-T_{n}^{2}\right)^{-1}$, we thus conclude

$$
a_{n}=\left\langle\hat{T}_{n} \Phi e_{n}^{+}, e_{n}^{+}\right\rangle=\left\langle T_{n}\left(I-T_{n}^{2}\right)^{-1} \Phi e_{n}^{+}, e_{n}^{+}\right\rangle
$$


On the other hand, all odd terms in the expansion of $b_{n}$ vanish, such that

$$
b_{n}^{ \pm}-\varphi_{2 n}^{ \pm}=\left\langle\left(\hat{T}_{n}-I\right) \Phi e_{n}^{\mp}, e_{n}^{ \pm}\right\rangle=\left\langle T_{n}^{2}\left(I-T_{n}^{2}\right)^{-1} \Phi e_{n}^{\mp}, e_{n}^{ \pm}\right\rangle
$$

We introduce the following notion for the sup-norm of a complex-valued function on a domain $U \subset \mathbb{C}$,

$$
|f|_{U}:=\sup _{\lambda \in U}|f(\lambda)|
$$

Lemma 3.6. If $\varphi \in H_{c}^{w}$ with $w \in \mathcal{M}^{1}$, then for any $\langle n\rangle \geqslant 8\|\varphi\|_{w}^{2}$ the coefficients $a_{n}$ and $b_{n}^{ \pm}$ are analytic functions on $\mathfrak{U}_{n}$ with bounds

$$
\left|a_{n}\right| \mathfrak{U}_{n} \leqslant \frac{1}{\langle n\rangle}\|\varphi\|_{w}^{2}, \quad w_{2 n}\left|b_{n}^{ \pm}-\varphi_{2 n}^{ \pm}\right| \mathfrak{U}_{n} \leqslant \frac{8}{\langle n\rangle}\|\varphi\|_{w}^{2}\left\|\varphi_{ \pm}\right\|_{w} .
$$

Proof. Since $\left\|T_{n}^{2}\right\|_{w ; n} \leqslant 1 / 2$, the series expansions of $a_{n}$ and $b_{n}^{ \pm}$converge uniformly on $\mathfrak{U}_{n}$ to analytic functions. Let $u=\left(I-T_{n}^{2}\right)^{-1} \Phi e_{n}^{+}$, then

$$
\|u\|_{w ; n} \leqslant\left\|\left(I-T_{n}^{2}\right)^{-1}\right\|_{w ; n}\left\|\Phi e_{n}^{+}\right\|_{w ; n} \leqslant 2\left\|\varphi_{-} e_{n}\right\|_{w ;-n}=2\left\|\varphi_{-}\right\|_{w},
$$

and with the series expansion $u=\sum_{m \in \mathbb{Z}} u_{m} e_{m}^{-}$we may write

$$
a_{n}=\left\langle T_{n} u, e_{n}^{+}\right\rangle=\sum_{m \neq n} \frac{\varphi_{n+m}^{-}}{\lambda-m \pi} u_{m}
$$

As $|n-m| \leqslant|n|$ implies $|n+m| \geqslant 2|n|-|n-m| \geqslant|n|$, this gives

$$
\left|a_{n}\right|_{\mathfrak{U}_{n}} \leqslant \sum_{m \neq n} \frac{1}{\langle n+m\rangle^{2}|n-m|} w_{n+m}\left|\varphi_{n+m}^{-}\right| \cdot w_{n+m}\left|u_{m}\right| \leqslant \frac{1}{\langle n\rangle}\left\|\varphi_{-}\right\| w\|u\|_{w ; n} \leqslant \frac{2}{\langle n\rangle}\left\|\varphi_{-}\right\|_{w}^{2} .
$$

Using the representation $a_{n}=\left\langle T_{n}\left(I-T_{n}^{2}\right) \Phi e_{n}^{-}, e_{n}^{-}\right\rangle$, we similarly obtain

$$
\left|a_{n}\right|_{\mathfrak{U}_{n}} \leqslant \frac{2}{\langle n\rangle}\left\|\varphi_{+}\right\|_{w}^{2} .
$$

Summing both estimates up gives the first bound. To obtain the second bound, we note that $b_{n}^{-}-\varphi_{2 n}^{-}=\left\langle T_{n}^{2} u, e_{n}^{-}\right\rangle$. Since $\left\langle f, e_{n}^{-}\right\rangle=\left\langle f e_{-n}, e_{2 n}^{-}\right\rangle$for any function $f \in L_{c}^{2}$, we conclude

$$
w_{2 n}\left|\left\langle T_{n}^{2} u, e_{n}^{-}\right\rangle\right| \leqslant\left\|T_{n}^{2} u\right\|_{w ; n} \leqslant \frac{8}{\langle n\rangle}\|\varphi\|_{w}^{2}\left\|\varphi_{-}\right\|_{w} .
$$

The proof for $b_{n}^{+}$is the same. 
In consequence, the determinant of $S_{n}$

$$
\operatorname{det} S_{n}=\left(\lambda-n \pi-a_{n}\right)^{2}-b_{n}^{+} b_{n}^{-}
$$

is analytic on $\mathfrak{U}_{n}$ and close to $(\lambda-n \pi)^{2}$ provided $\langle n\rangle$ is sufficiently large.

Lemma 3.7. Let $\varphi \in H_{c}^{1}$, then for any $\langle n\rangle \geqslant 8\|\varphi\|_{1}^{2}$, the determinant of $S_{n}$ has exactly two complex roots $\xi_{+}, \xi_{-}$in $\mathfrak{U}_{n}$, which are contained in the disk

$$
D_{n}:=\left\{|\lambda-\pi n| \leqslant \frac{\|\varphi\|_{1}^{2}}{\langle n\rangle}+\frac{\sqrt{2}\|\varphi\|_{1}}{\langle 2 n\rangle}\right\} \subset\left\{|\lambda-n \pi| \leqslant \frac{\pi}{5}\right\},
$$

and satisfy

$$
\left|\xi^{+}-\xi^{-}\right|^{2} \leqslant 6\left|b_{n}^{+} b_{n}^{-}\right|_{\mathfrak{U}_{n}}
$$

Proof. The estimates of the preceding lemma give for $\langle n\rangle \geqslant 8\|\varphi\|_{1}^{2}$

$$
\left|a_{n}\right| \mathfrak{U}_{n} \leqslant \frac{\|\varphi\|_{1}^{2}}{\langle n\rangle}, \quad\langle 2 n\rangle^{2}\left|b_{n}^{+} b_{n}^{-}\right|_{\mathfrak{U}_{n}} \leqslant\left(1+\frac{8\|\varphi\|_{1}}{\langle n\rangle}\right)^{2}\left\|\varphi_{+}\right\|_{1}\left\|\varphi_{-}\right\|_{1},
$$

where we used $w_{2 n}\left|b_{n}^{ \pm}\right| \leqslant\|\varphi\|_{w}+w_{2 n}\left|b_{n}^{ \pm}-\varphi_{2 n}^{ \pm}\right|$. Therefore,

$$
\left|a_{n}\right|_{\mathfrak{U}_{n}}+\left|b_{n}^{+} b_{n}^{-}\right|_{\mathfrak{U}_{n}}^{1 / 2} \leqslant \inf _{\lambda \in \mathfrak{U}_{n} \backslash D_{n}}|\lambda-n \pi|=\frac{\|\varphi\|_{1}^{2}}{\langle n\rangle}+\sqrt{2} \frac{\|\varphi\|_{1}}{\langle 2 n\rangle} \leqslant \pi / 5 .
$$

It follows from Rouche's Theorem that the function $h=\lambda-n \pi-a_{n}$ has a single root in $D_{n}$, just as $\lambda-n \pi$. Furthermore, $h^{2}$ and $\operatorname{det} S_{n}$ have the same number of roots in $D_{n}$, namely two when counted with multiplicity, while det $S_{n}$ clearly has no root in $\mathfrak{U}_{n} \backslash D_{n}$.

To estimate the distance of the roots, we write $\operatorname{det} S_{n}=g_{+} g_{-}$with

$$
g_{ \pm}=\lambda-\pi n-a_{n} \mp \sigma_{n}, \quad \sigma_{n}=\sqrt{b_{n}^{+} b_{n}^{-}},
$$

where the branch of the root is immaterial. Each root $\xi$ of $\operatorname{det} S_{n}$ is either a root of $g_{+}$or $g_{-}$, respectively, and thus satisfies $\xi=\pi n+a_{n}(\xi) \pm \sigma_{n}(\xi)$. Therefore,

$$
\left|\xi_{+}-\xi_{-}\right| \leqslant\left|a_{n}\left(\xi_{+}\right)-a_{n}\left(\xi_{-}\right)\right|+\left|\sigma_{n}\left(\xi_{+}\right) \pm \sigma_{n}\left(\xi_{-}\right)\right| \leqslant\left|\partial_{\lambda} a_{n}\right|_{D_{n}}\left|\xi_{+}-\xi_{-}\right|+2\left|\sigma_{n}\right| \mathfrak{U}_{n}
$$

Since $\operatorname{dist}\left(D_{n}, \partial \mathfrak{U}_{n}\right) \geqslant \pi / 2-\pi / 5$, the claim follows with Cauchy's estimate

$$
\left|\partial_{\lambda} a_{n}\right|_{D_{n}} \leqslant \frac{\left|a_{n}\right|_{\mathfrak{U}_{n}}}{\operatorname{dist}\left(D_{n}, \partial \mathfrak{U}_{n}\right)} \leqslant \frac{1 / 8}{\pi / 2-\pi / 5} \leqslant \frac{1}{6} .
$$

Proof of Theorem 3.1. For each $\langle n\rangle \geqslant 8\|\varphi\|_{1}^{2}$, Lemma 3.7 applies giving us the two roots $\xi_{+}$and $\xi_{-}$of det $S_{n}$ in $D_{n} \subset \mathfrak{U}_{n}$. As the strips $\mathfrak{U}_{n}$ cover the complex plane, and since $\lambda_{n}^{ \pm} \sim n \pi$ 
asymptotically as $n \rightarrow \pm \infty$ while there are no periodic eigenvalues in $\bigcup_{\langle n\rangle \geqslant 8\|\varphi\|_{1}^{2}}\left(\mathfrak{U}_{n} \backslash D_{n}\right)$, it follows by a standard counting argument that these roots have to be the periodic eigenvalues $\lambda_{n}^{ \pm}$. In turn, the remaining eigenvalues have to be contained in the strip

$$
\left\{\lambda \in \mathbb{C}:|\mathfrak{R} \lambda| \leqslant\left(8\|\varphi\|_{1}^{2}-1 / 2\right) \pi\right\} .
$$

To obtain the estimate for the imaginary part, suppose that $f$ is an $L_{c}^{2}$ normalized eigenfunction for $\lambda$, then

$$
2 \Im \lambda=\lambda-\bar{\lambda}=\langle L f, f\rangle-\langle f, L f\rangle=\left\langle\left(L-L^{*}\right) f, f\right\rangle .
$$

Further, using the $L^{\infty}$-estimate $\|g\|_{\infty} \leqslant \sqrt{2}\|g\|_{1}$ for $g \in H^{1}$, we find

$$
\left\|\left(L-L^{*}\right) f\right\|_{0} \leqslant \sqrt{2}\left\|\varphi_{+}-\overline{\varphi_{-}}\right\|_{1}\|f\|_{0} \leqslant 2\|\varphi\|_{1} .
$$

This completes the proof of the theorem.

Incidentally, we obtain the following estimate for the gap lengths, which we will use in Section 6.

Proposition 3.8. Suppose $\varphi \in H_{c}^{w}$ with $w \in \mathcal{M}^{1}$, then for any $\langle N\rangle \geqslant 8\|\varphi\|_{w}^{2}$,

$$
\sum_{|n| \geqslant N} w_{2 n}^{2}\left|\gamma_{n}(\varphi)\right|^{2} \leqslant 6\left\|R_{N} \varphi\right\|_{w}^{2}+\frac{1152}{\langle N\rangle}\|\varphi\|_{w}^{6},
$$

where $R_{N} \varphi=\sum_{|n| \geqslant N}\left(\varphi_{2 n}^{-} \mathrm{e}_{-2 n}, \varphi_{2 n}^{+} \mathrm{e}_{2 n}\right)$. If, in addition, $\varphi$ is in the complex neighborhood $W$ of $L_{r}^{2}$, then

$$
\sum_{n \in \mathbb{Z}} w_{2 n}^{2}\left|\gamma_{n}(\varphi)\right|^{2} \leqslant 265 \pi^{2} w^{2}\left[16\|\varphi\|_{w}^{2}\right]\left(1+\|\varphi\|_{w}^{2}\right)\|\varphi\|_{w}^{2} .
$$

Proof. By Lemma 3.7, we have for any $\langle n\rangle \geqslant 8\|\varphi\|_{w}^{2}$ the estimate

$$
\left|\gamma_{n}\right|^{2}=\left|\lambda_{n}^{+}-\lambda_{n}^{-}\right|^{2} \leqslant 6\left|b_{n}^{+} b_{n}^{-}\right|_{\mathfrak{U}_{n}} \leqslant 3\left(\left|b_{n}^{+}\right|_{\mathfrak{U}_{n}}^{2}+\left|b_{n}^{-}\right|_{\mathfrak{U}_{n}}^{2}\right) .
$$

Using $\left|b_{n}^{ \pm}{\mid \mathfrak{U}_{n}}_{n} \leqslant\right| \varphi_{2 n}^{ \pm}|+| b_{n}^{ \pm}-\left.\varphi_{2 n}^{ \pm}\right|_{\mathfrak{U}_{n}}$, we thus find for any $\langle N\rangle \geqslant 8\|\varphi\|_{w}^{2}$,

$$
\frac{1}{6} \sum_{|n| \geqslant N} w_{2 n}^{2}\left|\gamma_{n}(\varphi)\right|^{2} \leqslant \sum_{|n| \geqslant N} w_{2 n}^{2}\left(\left|\varphi_{2 n}^{+}\right|^{2}+\left|\varphi_{2 n}^{-}\right|^{2}+\left|b_{n}^{+}-\varphi_{2 n}^{+}\right|_{\mathfrak{U}_{n}}^{2}+\left|b_{n}^{-}-\varphi_{2 n}^{-}\right|_{\mathfrak{U}_{n}}^{2}\right) .
$$

Further by Lemma 3.6, $w_{2 n}^{2}\left|\varphi_{2 n}^{ \pm}-b_{n}^{ \pm}\right|_{\mathfrak{U}_{n}}^{2} \leqslant 64\langle n\rangle^{-2}\|\varphi\|_{w}^{4}\left\|\varphi_{ \pm}\right\|_{w}^{2}$, hence

$$
\frac{1}{6} \sum_{|n| \geqslant N} w_{2 n}^{2}\left|\gamma_{n}(\varphi)\right|^{2} \leqslant\left\|R_{N} \varphi\right\|_{w}^{2}+64\|\varphi\|_{w}^{6} \sum_{|n| \geqslant N} \frac{1}{\langle n\rangle^{2}},
$$

and the first claim follows with $\sum_{|n| \geqslant N} 1 /\langle n\rangle^{2} \leqslant 3 /\langle N\rangle$. 
If additionally $\varphi \in W$, then each gap is contained in its isolating neighborhood $U_{n}$. Those are disjoint complex disks centered on the real line, whose diameters for $|n|<N$ sum up to at most $(2 N-1) \pi$ by Theorem 3.1. Therefore,

$$
\sum_{|n|<N} w_{2 n}^{2}\left|\gamma_{n}(\varphi)\right|^{2} \leqslant w_{2 N-2}^{2}\left(\sum_{|n|<N}\left|\gamma_{n}(\varphi)\right|\right)^{2} \leqslant w_{2 N-2}^{2}(2 N-1)^{2} \pi^{2},
$$

and choosing $N+1 \geqslant 8\|\varphi\|_{w}^{2}>N$ gives the second claim.

If, for $\langle n\rangle \geqslant 8\|\varphi\|_{w}^{2}$, we use

$$
w_{2 n}\left|b_{n}^{ \pm}\right| \leqslant\left\|\varphi_{ \pm}\right\|_{w}+\frac{8}{\langle n\rangle}\|\varphi\|_{w}^{2}\left\|\varphi_{ \pm}\right\| \leqslant 2\left\|\varphi_{ \pm}\right\|_{w},
$$

then we obtain the individual gap estimate

$$
w_{2 n}\left|\gamma_{n}(\varphi)\right| \leqslant 4\|\varphi\|_{w}
$$

\section{Estimating the Actions}

As an immediate corollary to the localization obtained in the previous section, we obtain the following quantitative estimate of the high-level actions.

Proposition 4.1. If $\varphi \in H_{r}^{1}$, then for $\langle n\rangle \geqslant 8\|\varphi\|_{1}^{2}$ and $m \geqslant 0$,

$$
J_{n, 2 m+1}=\zeta_{n, m}^{2 m} I_{n}, \quad\left|\zeta_{n, m}-n \pi\right| \leqslant \frac{\|\varphi\|_{1}^{2}}{\langle n\rangle}+\frac{\sqrt{2}\|\varphi\|_{1}}{\langle 2 n\rangle}
$$

In particular, if $n \neq 0$ and $\langle n\rangle \geqslant 8\|\varphi\|_{1}^{2}$, then

$$
2^{-m}\langle 2 n \pi\rangle^{2 m} I_{n} \leqslant 4^{m} J_{n, 2 m+1} \leqslant\langle 2 n \pi\rangle^{2 m} I_{n}
$$

while the remaining actions for all $\langle n\rangle<8\|\varphi\|_{1}^{2}$ satisfy

$$
4^{m}\left|J_{n, 2 m+1}\right| \leqslant(16 \pi)^{2 m}\|\varphi\|_{1}^{4 m} I_{n}
$$

Proof. Recall from (8) that $J_{n, 2 m+1}=\zeta_{n, m}^{2 m} I_{n}$ with $\zeta_{n, m} \in\left[\lambda_{n}^{-}, \lambda_{n}^{+}\right]$. Provided $\langle n\rangle \geqslant 8\|\varphi\|_{1}^{2}$, the estimate of $\left|\zeta_{n, m}-n \pi\right|$ follows from Theorem 3.1. If additionally $n \neq 0$, then $\langle 2 n\rangle \geqslant 3\langle n\rangle / 2$ and hence $\left|\zeta_{n, m}-n \pi\right| \leqslant 1 / 2$. In consequence,

$$
\frac{1}{\sqrt{2}}\langle 2 n \pi\rangle \leqslant 2\left|\zeta_{n, m}\right| \leqslant\langle 2 n \pi\rangle, \quad n \neq 0 .
$$

Conversely, if $\langle n\rangle<8\|\varphi\|_{1}^{2}$, then $\left|\zeta_{n, m}\right| \leqslant 8 \pi\|\varphi\|_{1}^{2}$. 
In the sequel, we use Proposition 4.1 to obtain an estimate of

$$
\|I(\varphi)\|_{\ell_{2 m}^{1}}=\sum_{n \in \mathbb{Z}}\langle 2 n \pi\rangle^{2 m}\left|I_{n}\right|
$$

in terms of $\sum_{n \in \mathbb{Z}} J_{n, 2 m+1}$ and a remainder depending solely on $\|\varphi\|_{1}$. The trace formula and the polynomial structure of the Hamiltonians then allows us to obtain the first part of Theorem 1.2.

Lemma 4.2. For every $m \geqslant 1$,

$$
\|I(\varphi)\|_{\ell_{2 m}^{1}} \leqslant\langle 16 \pi\rangle^{2 m}\left(1+\|\varphi\|_{1}\right)^{4 m}\|\varphi\|_{0}^{2}+(-1)^{m+1} 2^{m} \mathcal{H}_{2 m+1}(\varphi),
$$

uniformly for all $\varphi \in H_{r}^{m}$.

Proof. Choose $N+1 \geqslant 8\|\varphi\|_{1}^{2}>N$, then by Proposition 4.1, the trace formula (3), and the positivity of the actions

$$
\sum_{|n|>N}\langle 2 n \pi\rangle^{2 m} I_{n} \leqslant 8^{m} \sum_{n \in \mathbb{Z}} J_{n, 2 m+1}=(-1)^{m+1} 2^{m} \mathcal{H}_{2 m+1} .
$$

On the other hand, by our choice of $N$ and the trace formula (2)

$$
\sum_{|n| \leqslant N}\langle 2 n \pi\rangle^{2 m} I_{n} \leqslant\langle 2 N \pi\rangle^{2 m} \sum_{n \in \mathbb{Z}} I_{n} \leqslant\langle 16 \pi\rangle^{2 m}\left(1+\|\varphi\|_{1}\right)^{4 m}\|\varphi\|_{0}^{2} .
$$

We denote the two components of $\varphi \in L_{r}^{2}$ by $\varphi=(\psi, \bar{\psi})$, and to simplify notation write $\psi_{(m)}=\partial_{x}^{m} \psi$ such that

$$
\int_{\mathbb{T}}\left|\psi_{(m)}\right|^{2} \mathrm{~d} x=\frac{1}{2}\left\|\varphi_{(m)}\right\|_{0}^{2}
$$

Proof of Theorem 1.2(i) for $m=1$. By the preceding lemma,

$$
\sum_{n \in \mathbb{Z}}\langle 2 n \pi\rangle^{2} I_{n} \leqslant\langle 16 \pi\rangle^{2}\left(1+\|\varphi\|_{1}\right)^{4}\|\varphi\|_{0}^{2}+2 \mathcal{H}_{3}(\varphi) .
$$

The Hamiltonian $\mathcal{H}_{3}(\varphi)=\int_{\mathbb{T}}\left(\left|\psi_{x}\right|^{2}+|\psi|^{4}\right) \mathrm{d} x$ may be estimated by

$$
\left|\mathcal{H}_{3}(\varphi)\right| \leqslant \frac{1}{2}\left\|\varphi_{x}\right\|_{0}^{2}+\frac{1}{2}\|\psi\|_{\infty}^{2}\|\varphi\|_{0}^{2} \leqslant \frac{1}{2}\left(1+\|\varphi\|_{0}^{2}\right)\|\varphi\|_{1}^{2},
$$

where we used the $L^{\infty}$-estimate $\|\psi\|_{\infty}^{2} \leqslant 2\|\psi\|_{1}^{2}=\|\varphi\|_{1}^{2}$. So we arrive at

$$
\|I(\varphi)\|_{\ell_{2}^{1}} \leqslant(32 \pi)^{2}\left(1+\|\varphi\|_{1}\right)^{4}\|\varphi\|_{1}^{2} .
$$


To proceed with the general case $m \geqslant 2$, we note that on $H_{r}^{m}$,

$$
(-1)^{m+1} \mathcal{H}_{2 m+1}(\varphi)=\int_{\mathbb{T}}\left(\left|\psi_{(m)}\right|^{2}+p_{2 m}\left(\psi, \bar{\psi}, \ldots, \psi_{(m-1)}, \bar{\psi}_{(m-1)}\right)\right) \mathrm{d} x,
$$

with $p_{2 m}$ being a homogenous polynomial of degree $2 m+2$ when $\psi, \bar{\psi}$, and $\partial_{x}$ each count as one degree. Further, the degree of each monomial of $p_{2 m}$ with respect to $\psi$ equals the degree with respect to $\bar{\psi}$-see Corollary A.4 from the appendix. Consequently, each monomial $\mathfrak{q}$ of $p_{2 m}$ may be estimated by

$$
|\mathfrak{q}| \leqslant C_{\mathfrak{q}}|\psi|^{\mu_{0}}\left|\psi_{X}\right|^{\mu_{1}} \cdots\left|\psi_{(m-1)}\right|^{\mu_{m-1}}
$$

with some positive constant $c_{\mathfrak{q}}$ and integers $\mu_{0}, \ldots, \mu_{m-1}$. It turns out to be convenient to use exponents which are multiples of 2 , that is,

$$
|\mathfrak{q}| \leqslant C_{\mathfrak{q}}|\psi|^{2 \sigma_{0}}\left|\psi_{X}\right|^{2 \sigma_{1}} \cdots\left|\psi_{(m-1)}\right|^{2 \sigma_{m-1}},
$$

with $\sigma_{i} \in \mathbb{Z}_{\geqslant 0} / 2$. Since $\mathfrak{q}$ has degree $2 m+2$, we have

$$
\sum_{0 \leqslant i \leqslant m-1} 2 \sigma_{i}(1+i)=2 m+2
$$

and as the degree with respect to $\psi$ and $\bar{\psi}$ is the same, $\sum_{0 \leqslant i \leqslant m-1} \sigma_{i}$ is an integer even though $\sigma_{i} \in \mathbb{Z}_{\geqslant 0} / 2$. Denote by $\mathcal{I}_{2 m+2} \subset\left(\mathbb{Z}_{\geqslant 0} / 2\right)^{m}$ the set of all multi-indices $\sigma=\left(\sigma_{i}\right)_{0 \leqslant i \leqslant m-1}$ satisfying the constraints

$$
\sum_{0 \leqslant i \leqslant m-1} 2(1+i) \sigma_{i}=2 m+2, \quad|\sigma|:=\sum_{0 \leqslant i \leqslant m-1} \sigma_{i} \in \mathbb{Z}
$$

Then, we obtain the estimate

$$
\left|p_{2 m}\right| \leqslant P_{2 m}, \quad P_{2 m}:=\sum_{\sigma \in \mathcal{I}_{2 m+2}} C_{\sigma}|\psi|^{2 \sigma_{0}}\left|\psi_{X}\right|^{2 \sigma_{1}} \cdots\left|\psi_{(m-1)}\right|^{2 \sigma_{m-1}},
$$

with positive constants $C_{\sigma}$. The majorant $P_{2 m}$ allows us to obtain detailed estimates of $p_{2 m}$ in Section 5. For now, we content ourselves with the following preliminary result.

Proof of Theorem 1.2(i) for $m \geqslant 2$. If $\varphi \in H_{r}^{m}$ and $\sigma \in \mathcal{I}_{2 m+2}$, then by (10)

$$
2 m+2 \geqslant 2 m \sigma_{m-1} .
$$

Suppose $\sigma_{m-1}>1$, then necessarily $m=2$ and $\sigma_{m-1}=\frac{3}{2}$ while all other indices $\sigma_{i}$ vanish. In this case, however, $|\sigma|=\sigma_{m-1}$ is not an integer, giving a contradiction. Therefore, $\sigma_{m-1} \leqslant 1$ for all $\sigma \in \mathcal{I}_{2 m+1}$, that is, $P_{2 m}$ is at most quadratic in $\left|\psi_{(m-1)}\right|$. With 
$\left\|\psi_{(m-1)}\right\|_{L^{1}} \leqslant\left\|\psi_{(m-1)}\right\|_{0}$, it thus follows

$$
\int_{\mathbb{T}}\left|\psi_{(m-1)}^{2 \sigma_{m-1}}\right| \leqslant \frac{1}{2}\left\|\varphi_{(m-1)}\right\|_{0}^{2 \sigma_{m-1}},
$$

and by estimating the remaining factors using the $L^{\infty}$-estimate

$$
\left|\psi_{(j)}^{2 \sigma_{j}}\right| \leqslant\left\|\psi_{(j)}\right\|_{\infty}^{2 \sigma_{j}} \leqslant\|\varphi\|_{j+1}^{2 \sigma_{j}},
$$

we arrive at

$$
\int_{\mathbb{T}}\left|p_{2 m}\right| \mathrm{d} x \leqslant \int_{\mathbb{T}} P_{2 m} \mathrm{~d} x \leqslant \sum_{\mathcal{I}_{2 m+2}} C_{\sigma}\|\varphi\|_{m-1}^{2 \sigma_{0}+\cdots+2 \sigma_{m-1}}
$$

Since $2 \sigma_{0}+\cdots+2 \sigma_{m-1} \leqslant 2 m+2$, we obtain for the Hamiltonian

$$
\left|\mathcal{H}_{2 m+1}(\varphi)\right| \leqslant \frac{1}{2}\|\varphi\|_{m}^{2}+b_{m}^{2}\left(1+\|\varphi\|_{m-1}^{2 m}\right)\|\varphi\|_{m-1}^{2},
$$

with some positive constant $b_{m}$. Together with Lemma 4.2 , this gives

$$
\|I(\varphi)\|_{\ell_{2 m}^{1}} \leqslant 2^{m-1}\|\varphi\|_{m}^{2}+c_{m}^{2}\left(1+\|\varphi\|_{m-1}\right)^{4 m}\|\varphi\|_{m-1}^{2}, \quad m \geqslant 2 .
$$

\section{Estimating the Sobolev Norms}

We now turn to the converse problem of estimating the Sobolev norms of the potential in terms of weighted norms of its actions on level one. Our starting point is the identity

$$
\frac{1}{2}\left\|\varphi_{(m)}\right\|_{0}^{2}=4^{m} \sum_{n \in \mathbb{Z}} J_{n, 2 m+1}-\int_{\mathbb{T}} p_{2 m} \mathrm{~d} x, \quad m \geqslant 1,
$$

which is obtained by combining Corollary A.4 and the trace formula (3). The key step is estimating the actions $J_{n, 2 m+1}$ in terms of $I_{n}$. Subsequently, we invoke an inductive argument using that $p_{2 m}$ contains only derivatives up to order $m-1$. The main difficulty is to estimate the actions $J_{n, 2 m+1}$ below the threshold of $\langle n\rangle$ provided by Proposition 4.1. Even though there are only finitely many of them, they cannot be controlled by the $L^{2}$-norm $\|\varphi\|_{0}$ as one can translate the spectrum of $\varphi$ without changing $\|\varphi\|_{0}$. Instead, we use the $H^{1}$-norm $\|\varphi\|_{1}$, and provide estimates of $\|\varphi\|_{1}$ in terms of $I_{n}$ by separate arguments which were inspired by work of Korotyaev [19] and Korotyaev and Kargaev [21].

Lemma 5.1. Uniformly for all $\varphi \in H_{r}^{1}$,

$$
\mathcal{H}_{3}(\varphi)-2 \mathcal{H}_{1}^{2}(\varphi) \leqslant \sum_{n \in \mathbb{Z}}(2 n \pi)^{2} I_{n}(\varphi)
$$


In particular, $\mathcal{H}_{3}(\varphi) \leqslant\|I(\varphi)\|_{\ell_{2}^{1}}+2\|I(\varphi)\|_{\ell^{1}}^{2}$ and

$$
\frac{1}{3}\|\varphi\|_{1}^{2} \leqslant\|I(\varphi)\|_{\ell_{2}^{1}}+\|I(\varphi)\|_{\ell^{1}}^{2} .
$$

Proof. As the Hamiltonians and the actions are continuous on $H_{r}^{1}$, it suffices to consider the case of a finite gap potential. Let $C_{r}$ denote a sufficiently large circle enclosing all open gaps of $\varphi$, then the primitive $F$ of $\omega$ defined in Section 2 is analytic outside $C_{r}$ and its Laurent expansion is given by Lemma 2.4. Thus, by the Residue Theorem,

$$
\frac{1}{\pi} \int_{C_{r}} F^{3}(\lambda) \mathrm{d} \lambda=\frac{3}{8 \mathrm{i} \pi} \int_{C_{r}} \frac{1}{\lambda}\left(\mathcal{H}_{3}-2 \mathcal{H}_{1}^{2}\right) \mathrm{d} \lambda=\frac{3}{4}\left(\mathcal{H}_{3}-2 \mathcal{H}_{1}^{2}\right) .
$$

The right-hand side is real as $\varphi$ is of real type, and

$$
\Re \int_{C_{r}} F^{3}(\lambda) \mathrm{d} \lambda=\sum_{n \in \mathbb{Z}} \int_{\lambda_{n}^{-}}^{\lambda_{n}^{+}} \Re\left(F^{3}(\lambda-\mathrm{i} 0)-F^{3}(\lambda+\mathrm{i} 0)\right) \mathrm{d} \lambda .
$$

Furthermore, by Lemma 2.3 we have for $\lambda_{n}^{-}<\lambda<\lambda_{n}^{+}$,

$$
\mathfrak{R}\left(F^{3}(\lambda-\mathrm{i} 0)-F^{3}(\lambda+\mathrm{i} 0)\right)=-2 f_{n}^{3}(\lambda)+6(n \pi)^{2} f_{n}(\lambda),
$$

and since $f_{n}$ is nonnegative, we conclude with (7)

$$
\frac{1}{\pi} \int_{C_{r}} F^{3}(\lambda) \mathrm{d} \lambda \leqslant \sum_{n \in \mathbb{Z}} \frac{6}{\pi} \int_{\lambda_{n}^{-}}^{\lambda_{n}^{+}}(n \pi)^{2} f_{n}(\lambda) \mathrm{d} \lambda=\frac{3}{4} \sum_{n \in \mathbb{Z}}(2 n \pi)^{2} I_{n} .
$$

This proves the first claim. Note that $\langle 2 n \pi\rangle^{2} \leqslant \frac{3}{2}\left(1+(2 n \pi)^{2}\right)$ for $n \in \mathbb{Z}$, so

$$
\frac{1}{3}\|\varphi\|_{1}^{2} \leqslant \frac{1}{2}\left(\left\|\varphi_{X}\right\|_{0}^{2}+\|\varphi\|_{0}^{2}\right)=\mathcal{H}_{3}-\int_{\mathbb{T}}|\psi|^{4} \mathrm{~d} x+\mathcal{H}_{1} .
$$

Since $\int_{\mathbb{T}}|\psi|^{4} \mathrm{~d} x \geqslant \mathcal{H}_{1}^{2}(\varphi)$, the second claim follows with

$$
\frac{1}{3}\|\varphi\|_{1}^{2} \leqslant \mathcal{H}_{3}-2 \mathcal{H}_{1}^{2}+\mathcal{H}_{1}^{2}+\mathcal{H}_{1} \leqslant\|I(\varphi)\|_{\ell_{2}^{1}}+\|I(\varphi)\|_{\ell^{1}}^{2}
$$

Proof of Theorem 1.2(ii). The case $m=1$ is an immediate corollary of the lemma above. For the case $m \geqslant 2$, we find with (12) and Lemma 5.2

$$
\frac{1}{2}\left\|\varphi_{(m)}\right\|_{0}^{2}=\sum_{n \in \mathbb{Z}} 4^{m} J_{n, 2 m+1}-\int_{\mathbb{T}} p_{2 m} \mathrm{~d} x \leqslant\|I\|_{\ell_{2 m}^{1}}+c_{m}\left(1+\|I\|_{\ell_{2 m-2}^{1}}\right)^{4 m-3}\|I\|_{\ell_{2 m-2}^{1}}+\int_{\mathbb{T}}\left|p_{2 m}\right| \mathrm{d} x .
$$

It remains to consider the $p_{2 m}$-term, for which we have the estimate (11)

$$
\int_{\mathbb{T}}\left|p_{2 m}\right| \mathrm{d} x \leqslant \int_{\mathbb{T}} P_{2 m} \mathrm{~d} x=\sum_{\mathcal{I}_{2 m+2}} \int_{\mathbb{T}} C_{\sigma}|\psi|^{2 \sigma_{0}} \cdots\left|\psi_{(m-1)}\right|^{2 \sigma_{m-1}} \mathrm{~d} x
$$


The right-hand side only contains derivatives of $\psi$ up to order $m-1$ and the exponents $\sigma_{i}$ satisfy the constraints (10). We use this to prove by induction for any $m \geqslant 2$

$$
\frac{1}{2}\left\|\varphi_{(m)}\right\|_{0}^{2} \leqslant\|I\|_{\ell_{2 m}^{1}}+d_{m}^{2}\left(1+\|I\|_{\ell_{2 m-2}^{1}}\right)^{\alpha_{m}}\|I\|_{\ell_{2 m-2}^{1}},
$$

with absolute constants $d_{m}$ and exponents $\alpha_{m}:=4 m-3$. By setting $\alpha_{0}=0$ and $\alpha_{1}=1$, this already holds for $m=0,1$.

Now consider the inductive step $m-1 \mapsto m$. Recall from the proof of Theorem 1.2(i) that $P_{2 m}$ is at most quadratic in $\left|\psi_{(m-1)}\right|$ for $m \geqslant 2$. Thus, we have the following expansion:

$$
\begin{aligned}
P_{2 m}= & P_{2 m ; 2}\left(|\psi|, \ldots,\left|\psi_{(m-2)}\right|\right)\left|\psi_{(m-1)}\right|^{2}+P_{2 m ; 1}\left(|\psi|, \ldots,\left|\psi_{(m-2)}\right|\right)\left|\psi_{(m-1)}\right| \\
& +P_{2 m ; 0}\left(|\psi|, \ldots,\left|\psi_{(m-2)}\right|\right)
\end{aligned}
$$

where for $0 \leqslant k \leqslant 2$ the entity $P_{2 m ; k}\left|\psi_{(m-1)}\right|^{k}$ incorporates those $\sigma \in \mathcal{I}_{2 m+2}$ with $2 \sigma_{m-1}=k$. In particular, $P_{2 m ; 2}=c|\psi|^{2}$ with some $c \geqslant 0$. The three terms in the expansion of $P_{2 m}$ will be estimated separately.

Let us begin with $P_{2 m ; 2}\left|\psi_{(m-1)}\right|^{2}=c|\psi|^{2}\left|\psi_{(m-1)}\right|^{2}$. To avoid a flood a constants, we write $a \lessdot b$ if $a \leqslant c \cdot b$ with an absolute constant $c$ which is independent of $\psi$ but may depend on $m$. Using the $L^{\infty}$-estimate and the induction hypothesis for $m-1$, we get

$$
\int_{\mathbb{T}} P_{2 m ; 2}\left|\psi_{(m-1)}\right|^{2} \mathrm{~d} x \lessdot\|\psi\|_{\infty}^{2}\left\|\varphi_{(m-1)}\right\|_{0}^{2} \lessdot\|\varphi\|_{1}^{2}\left\|\varphi_{(m-1)}\right\|_{0}^{2} \lessdot\left(1+\|I\|_{\ell_{2 m-2}^{1}}\right)^{\alpha_{m-1}+2}\|I\|_{\ell_{2 m-2}^{1}},
$$

where $\alpha_{m-1}+2=4(m-1)-1 \leqslant 4 m-3=\alpha_{m}$.

Next, consider the term $P_{2 m ; 1}\left|\psi_{(m-1)}\right|$ which involves all multi-indices $\sigma=$ $\left(\sigma_{i}\right)_{0 \leqslant i \leqslant m-1}$ with $2 \sigma_{m-1}=1$. Recall that $|\sigma|=\sum_{0 \leqslant i \leqslant m-1} \sigma_{i}$ is an integer, hence there has to be an index $\sigma_{j} \neq 0$ with $0 \leqslant j \leqslant m-2$. By Cauchy-Schwarz and the $L^{\infty}$-estimate

$$
\begin{aligned}
\int_{\mathbb{T}}\left|\psi_{(j)}\right|^{2 \sigma_{j}}\left|\psi_{(m-1)}\right| \mathrm{d} x & \lessdot\|\varphi\|_{j+1}^{2 \sigma_{j}-1}\left\|\varphi_{(j)}\right\|_{0}\left\|\varphi_{(m-1)}\right\|_{0} \\
& \lessdot\left(1+\|I\|_{\ell_{2 m-2}^{1}}\right)^{\alpha_{j+1}\left(\sigma_{j}-1 / 2\right)+\alpha_{j} / 2+\alpha_{m-1} / 2}\|I\|_{\ell_{2 m-2}^{1}}^{\sigma_{j}+1 / 2} \\
& \lessdot\left(1+\|I\|_{\ell_{2 m-2}^{1}}\right)^{\alpha_{j+1} \sigma_{j}+\alpha_{m} \sigma_{m-1}-\delta_{j}-\delta_{m-1}}\|I\|_{\ell_{2 m-2}^{1}}^{\sigma_{j}+\sigma_{m-1}},
\end{aligned}
$$

where we have set $\delta_{k}:=\left(\alpha_{k+1}-\alpha_{k}\right) / 2$, so $\delta_{0}=\frac{1}{2}$ and $\delta_{k}=2$ for all $k \geqslant 1$. The remaining factors are estimated by

$$
\left|\psi_{(k)}\right|^{2 \sigma_{k}} \leqslant\|\varphi\|_{k+1}^{2 \sigma_{k}} \lessdot\left(1+\|I\|_{\ell_{2 m-2}^{1}}\right)^{\alpha_{k+1} \sigma_{k}}\|I\|_{\ell_{2 m-2}^{1}}^{\sigma_{k}} .
$$


Both estimates together yield

$$
\begin{aligned}
& \int_{\mathbb{T}}|\psi|^{2 \sigma_{0}} \cdots\left|\psi_{(m-1)}\right|^{2 \sigma_{m-1}} \mathrm{~d} x \lessdot\left(1+\|I\|_{\ell_{2 m-2}^{1}}\right)^{\alpha_{1} \sigma_{0}+\cdots+\alpha_{m} \sigma_{m-1}-\delta_{j}-\delta_{m-1}}\|I\|_{\ell_{2 m-2}^{1}}^{\sigma_{0}+\cdots+\sigma_{m-1}} \\
& \lessdot\left(1+\|I\|_{\ell_{2 m-2}^{1}}\right)^{\left(\alpha_{1}+1\right) \sigma_{0}+\cdots+\left(\alpha_{m}+1\right) \sigma_{m-1}-\delta_{j}-\delta_{m-1}-1}\|I\|_{\ell_{2 m-2}^{1}} .
\end{aligned}
$$

As $\delta_{j}+\delta_{m-1} \geqslant \frac{5}{2}$ and $\sum_{0 \leqslant i \leqslant m-1}(1+i) \sigma_{i}=m+1$, we find for the exponent

$$
\sum_{0 \leqslant i \leqslant m-1}\left(\alpha_{i+1}+1\right) \sigma_{i}-\delta_{j}-\delta_{m-1}-1 \leqslant 4 m-2|\sigma|+1 / 2
$$

If we had $|\sigma|=1$, then

$$
m+1=\sum_{0 \leqslant i \leqslant m-1}(1+i) \sigma_{i} \leqslant \frac{m}{2}+\frac{m-1}{2},
$$

which is impossible, thus $|\sigma| \geqslant 2$. Consequently, the exponent is not greater than $\alpha_{m}=$ $4 m-3$ and hence

$$
\int_{\mathbb{T}} P_{2 m ; 1}\left|\psi_{(m-1)}\right| \mathrm{d} x \lessdot\left(1+\|I\|_{\ell_{2 m-2}^{1}}\right)^{\alpha_{m}}\|I\|_{\ell_{2 m-2}^{1}} .
$$

It remains to estimate the term $P_{2 m ; 0}$ involving all $\sigma=\left(\sigma_{i}\right)_{0 \leqslant i \leqslant m-1}$ with $\sigma_{m-1}=0$. We first consider those multi-indices $\sigma$ with $\sigma_{i}, \sigma_{j} \neq 0$ for some $i<j \leqslant m-2$. In that case, by Cauchy-Schwarz,

$$
\begin{aligned}
\int_{\mathbb{T}}\left|\psi_{(i)}\right|^{2 \sigma_{i}}\left|\psi_{(j)}\right|^{2 \sigma_{j}} \mathrm{~d} x & \lessdot\|\varphi\|_{i+1}^{2 \sigma_{i}-1}\|\varphi\|_{j+1}^{2 \sigma_{j}-1}\left\|\varphi_{(i)}\right\|_{0}\left\|\varphi_{(j)}\right\|_{0} \\
& \lessdot\left(1+\|I\|_{\ell_{2 m-2}^{1}}\right)^{\alpha_{i+1} \sigma_{i}+\alpha_{j+1} \sigma_{j}-\delta_{i}-\delta_{j}}\|I\|_{\ell_{2 m-2}^{1}}^{\sigma_{i}+\sigma_{j}}
\end{aligned}
$$

while the remaining factors are estimated as usual,

$$
\left|\psi_{(k)}\right|^{2 \sigma_{k}} \lessdot\|\varphi\|_{k+1}^{2 \sigma_{k}} \lessdot\left(1+\|I\|_{\ell_{2 m-2}^{1}}\right)^{\alpha_{k+1} \sigma_{k}}\|I\|_{\ell_{2 m-2}^{1}}^{\sigma_{k}}
$$

Together, both estimates give

$$
\begin{aligned}
& \int_{\mathbb{T}}|\psi|^{2 \sigma_{0}} \cdots\left|\psi_{(m-2)}\right|^{2 \sigma_{m-2}} \mathrm{~d} x \lessdot\left(1+\|I\|_{\ell_{2 m-2}^{1}}\right)^{\alpha_{1} \sigma_{0}+\alpha_{2} \sigma_{1}+\cdots+\alpha_{m-1} \sigma_{m-2}-\delta_{i}-\delta_{j}}\|I\|_{\ell_{2 m-2}^{1}}^{\sigma_{0}+\cdots+\sigma_{m-2}} \\
& \lessdot\left(1+\|I\|_{\ell_{2 m-2}^{1}}\right)^{\left(\alpha_{1}+1\right) \sigma_{0}+\cdots+\left(\alpha_{m-1}+1\right) \sigma_{m-2}-\delta_{i}-\delta_{j}-1}\|I\|_{\ell_{2 m-2}^{1}} .
\end{aligned}
$$

As before $\delta_{i}+\delta_{j} \geqslant 5 / 2$ and $|\sigma| \geqslant 2$, hence the exponent is not greater than $\alpha_{m}=4 m-3$. 
Finally, consider the case of a monomial $c \psi_{(i)}^{2 \sigma_{i}}$, that is, $(1+i) \sigma_{i}=m+1$ for some $0 \leqslant i \leqslant m-2$. If $i=0$, then $\sigma_{0}=m+1$ and

$$
\int_{\mathbb{T}}|\psi|^{2 m+2} \mathrm{~d} x \lessdot\|\varphi\|_{1}^{2 m}\|\varphi\|_{0}^{2} \lessdot\left(1+\|I\|_{\ell_{2}^{1}}\right)^{2 m}\|I\|_{\ell^{1}},
$$

with $2 m \leqslant \alpha_{m}$. Conversely, if $i \geqslant 1$, as necessarily $2 \sigma_{i} \geqslant 2$, we find

$$
\begin{aligned}
\int_{\mathbb{T}}\left|\psi_{(i)}\right|^{2 \sigma_{i}} \mathrm{~d} x \lessdot\|\varphi\|_{i+1}^{2 \sigma_{i}-2}\left\|\varphi_{(i)}\right\|_{0}^{2} & \lessdot\left(1+\|I\|_{\ell_{2 m-2}^{1}}\right)^{\alpha_{i+1}\left(\sigma_{i}-1\right)+\alpha_{i}}\|I\|_{\ell_{2 m-2}^{1}}^{\sigma_{i}} \\
& \lessdot\left(1+\|I\|_{\ell_{2 m-2}^{1}}\right)^{\left(\alpha_{i+1}+1\right) \sigma_{i}-5}\|I\|_{\ell_{2 m-2}^{1}},
\end{aligned}
$$

and further

$$
\left(\alpha_{i+1}+1\right) \sigma_{i}-5=4(m+1)-5-2 \sigma_{i} \leqslant \alpha_{m} .
$$

Altogether we thus obtain

$$
\int_{\mathbb{T}} P_{2 m} \mathrm{~d} x \lessdot\left(1+\|I\|_{\ell_{2 m-2}^{1}}\right)^{\alpha_{m}}\|I\|_{\ell_{2 m-2}^{1}},
$$

which completes the induction step and proves Theorem 1.2(ii).

Lemma 5.2. For each $m \geqslant 1$,

$$
\sum_{n \in \mathbb{Z}} 4^{m} J_{n, 2 m+1} \leqslant\|I(\varphi)\|_{\ell_{2 m}^{1}}+(64 \pi)^{2 m-2}\left(1+\|I(\varphi)\|_{\ell_{2}^{1}}\right)^{4 m-3}\|I(\varphi)\|_{\ell_{2}^{1}},
$$

uniformly for all $\varphi \in H_{r}^{m}$.

Proof. Let $N+1 \geqslant 8\|\varphi\|_{1}^{2}>N$, then by Proposition 4.1

$$
\sum_{|n|>N} 4^{m} J_{n, 2 m+1} \leqslant \sum_{|n|>N}\langle 2 n \pi\rangle^{2 m} I_{n}
$$

while for the remaining actions $J_{n, 2 m+1}=\tilde{\zeta}_{n, m}^{2 m-2} J_{n, 3}$ and hence

$$
\begin{aligned}
\sum_{|n| \leqslant N} 4^{m} J_{n, 2 m+1} & \leqslant(16 \pi)^{2 m-2}\left(1+\|\varphi\|_{1}^{2}\right)^{2 m-2} \sum_{|n| \leqslant N} 4 J_{n, 3} \\
& \leqslant(64 \pi)^{2 m-2}\left(1+\|I(\varphi)\|_{\ell_{2}^{1}}\right)^{4 m-4} \sum_{|n| \leqslant N} 4 J_{n, 3} .
\end{aligned}
$$

By the trace formula (3) and Lemma 5.1, we finally obtain

$$
\sum_{n \in \mathbb{Z}} 4 J_{n, 3}=\mathcal{H}_{3} \leqslant\|I(\varphi)\|_{\ell_{2}^{1}}+2\|I(\varphi)\|_{\ell^{1}}^{2}
$$




\section{Actions, Weighted Sobolev Spaces, and Gap Lengths}

The case of estimating the action variables of $\varphi$ in standard Sobolev spaces $H_{r}^{m}$ with integers $m \geqslant 1$ is somewhat special due to the presence of the trace formula (3). When arbitrary weighted Sobolev spaces $H_{r}^{w}$ are considered, there is no identity known to exist relating $\|\varphi\|_{w}$ to Hamiltonians of the NLS hierarchy. Albeit, even in the case of weighted Sobolev spaces, the regularity properties of $\varphi$ are well known to be closely related to the decay properties of the gap lengths $\gamma_{n}(\varphi)$-see, for example, $[7,16]$ and Section 3. Moreover,

$$
\frac{4 I_{n}}{\gamma_{n}^{2}}=1+\ell_{n}^{2}
$$

is known to hold locally uniformly on $L_{r}^{2}$ and hence uniformly on bounded subsets of $H_{r}^{1}$ - see [10]. In this section, we prove a quantitative version of (13) which is quadratic in $\|\varphi\|_{1}$ on all of $H_{r}^{1}$. From this and the estimates of the gap lengths given in Section 3, we then obtain Theorem 1.3.

To set the stage, let $\varphi \in W$ and recall from (6),

$$
I_{n}=\frac{1}{\pi} \int_{\Gamma_{n}} \lambda \omega=-\frac{1}{\pi} \int_{\Gamma_{n}}\left(\lambda_{n}^{\bullet}-\lambda\right) \omega .
$$

Here the latter identity follows as $\omega$ is closed around the gap. In the case $I_{n} \neq 0$, or equivalently $\gamma_{n} \neq 0$, we shrink the contour $\Gamma_{n}$ to the straight line $\left[\lambda_{n}^{-}, \lambda_{n}^{+}\right]$and insert the product representation (4) of $\omega$, to obtain

$$
I_{n}=-\frac{2}{\pi} \int_{\lambda_{n}^{-}}^{\lambda_{n}^{+}} \frac{\left(\lambda_{n}^{\bullet}-\lambda\right)^{2}}{i \zeta_{n}(\lambda)} \chi_{n}(\lambda) \mathrm{d} \lambda, \quad \chi_{n}(\lambda)=\prod_{n \neq m} \frac{\lambda_{m}^{\bullet}-\lambda}{\zeta_{m}(\lambda)} .
$$

It follows with the substitution $\lambda=\tau_{n}+t \gamma_{n} / 2$ that

$$
\frac{4 I_{n}}{\gamma_{n}^{2}}=\frac{2}{\pi} \int_{-1}^{1} \frac{\left(t-t_{n}\right)^{2}}{\sqrt[+]{1-t^{2}}} \chi_{n}\left(\tau_{n}+t \gamma_{n} / 2\right) \mathrm{d} t, \quad t_{n}=2\left(\lambda_{n}^{\bullet}-\tau_{n}\right) / \gamma_{n}
$$

By Lemma A.1, there exists an open connected neighborhood $\hat{W} \subset W$ of $L_{r}^{2}$ such that $\left|\lambda_{m}^{\bullet}-\tau_{m}\right| \leqslant\left|\gamma_{m}\right|$ for all $m \in \mathbb{Z}$, hence $\left|t_{n}\right| \leqslant 2$, and thus

$$
\left|\frac{4 I_{n}}{\gamma_{n}^{2}}\right| \leqslant 9\left|\chi_{n}\right|_{\left[\lambda_{n}^{-}, \lambda_{n}^{+}\right]}
$$

To get a quantitative version of (13), we thus need a uniform estimate of $\chi_{n}$. 
Lemma 6.1. On $H_{c}^{1} \cap \hat{W}$ for any $|n| \geqslant\langle N\rangle \geqslant 8\|\varphi\|_{1}^{2}$,

$$
\left|\chi_{n}\right|_{\left[\lambda_{\bar{n}}, \lambda_{n}^{+}\right]} \leqslant \mathrm{e}^{2}\left(\frac{|n|+N+3 / 5}{|n|-N-3 / 5}\right) \leqslant 2^{9}\left(1+\|\varphi\|_{1}^{2}\right) .
$$

Proof. Suppose $\varphi \in H_{c}^{1} \cap \hat{W}$ and choose $\langle N\rangle \geqslant 8\|\varphi\|_{1}^{2}>N$. For $|n| \geqslant N$, we split the product $\chi_{n}$ into two parts,

$$
\chi_{n}(\lambda)=\left(\prod_{|m| \leqslant N} \frac{\lambda_{m}^{\bullet}-\lambda}{\varsigma_{m}(\lambda)}\right)\left(\prod_{\substack{n \neq m \\|m|>N}} \frac{\lambda_{m}^{\bullet}-\lambda}{\varsigma_{m}(\lambda)}\right)
$$

If $|k|>N$, then by Theorem 3.1

$$
\left|\lambda_{k}^{ \pm}-k \pi\right| \leqslant \frac{\|\varphi\|_{1}^{2}}{\langle k\rangle}+\frac{\sqrt{2}\|\varphi\|_{1}}{\langle 2 k\rangle} \leqslant \pi / 8,
$$

where we used that $\langle 2 k\rangle \geqslant 3\langle k\rangle / 2$. Thus, for $|m|,|n|>N$ and $\lambda \in\left[\lambda_{n}^{-}, \lambda_{n}^{+}\right]$,

$$
\left|\tau_{m}-\lambda\right| \geqslant 2|n-m|
$$

Further, $\left|\gamma_{m}\right| \leqslant 4\|\varphi\|_{1} /\langle 2 m\rangle$ by the individual gap estimate (9), hence

$$
\left|\frac{\gamma_{m} / 2}{\tau_{m}-\lambda}\right| \leqslant \frac{\|\varphi\|_{1}}{\langle 2 m\rangle|n-m|} \leqslant 1 / 4 .
$$

It follows that $\left|\varsigma_{m}(\lambda)\right| \geqslant\left|\tau_{m}-\lambda\right|-\left|\gamma_{m}\right| / 2$. Moreover, $\left|\lambda_{m}^{\bullet}-\tau_{m}\right| \leqslant\left|\gamma_{m}\right|$ as $\varphi \in \hat{W}$, thus with $(1+2 r) /(1-r) \leqslant 1+4 r$ for $0 \leqslant r \leqslant \frac{1}{4}$, we conclude

$$
\left|\frac{\lambda_{m}^{\bullet}-\lambda}{\zeta_{m}(\lambda)}\right| \leqslant \frac{\left|\tau_{m}-\lambda\right|+\left|\gamma_{m}\right|}{\left|\tau_{m}-\lambda\right|-\left|\gamma_{m}\right| / 2} \leqslant 1+\frac{\left|\gamma_{m}\right|}{|n-m|} .
$$

It follows with Cauchy-Schwarz that

$$
\begin{aligned}
\sum_{\substack{m \neq n \\
|m|>N}} \frac{\left|\gamma_{m}\right|}{|n-m|} & \leqslant\left(\sum_{\substack{m \neq n \\
|m|>N}} \frac{1}{\langle 2 m\rangle^{2}|n-m|^{2}}\right)^{1 / 2}\left(\sum_{|m|>N}\langle 2 m\rangle^{2}\left|\gamma_{m}\right|^{2}\right)^{1 / 2} \\
& \leqslant \frac{2}{\langle 2 N+2\rangle}\left(\sum_{|m|>N}\langle 2 m\rangle^{2}\left|\gamma_{m}\right|^{2}\right)^{1 / 2},
\end{aligned}
$$

and by Proposition 3.8

$$
\sum_{|m|>N}\langle 2 m\rangle^{2}\left|\gamma_{m}\right|^{2} \leqslant 6\left\|\mathcal{R}_{N} \varphi\right\|_{1}^{2}+144\|\varphi\|_{1}^{4} \leqslant 4\langle N\rangle^{2} .
$$


Therefore, by the standard estimates for infinite products,

$$
\prod_{\substack{|m|>N \\ m \neq n}}\left|\frac{\lambda_{m}^{\bullet}-\lambda}{S_{m}(\lambda)}\right| \leqslant \exp (4\langle N\rangle /\langle 2 N+2\rangle) \leqslant \mathrm{e}^{2}
$$

To estimate the remaining part of the product, we note that $\lambda_{m}^{\bullet}$ and $\lambda_{m}^{ \pm}$are contained in the isolating neighborhood $U_{m}$, which is a complex disk centered on the real line. Thus, if $\lambda \in\left[\lambda_{n}^{-}, \lambda_{n}^{+}\right]$and $n>N$, then

$$
\left|\lambda_{m-1}^{ \pm}-\lambda\right|>\left|\lambda_{m}^{\bullet}-\lambda\right|, \quad m \leqslant N
$$

and consequently

$$
\prod_{|m| \leqslant N}\left|\frac{\lambda_{m}^{\bullet}-\lambda}{\varsigma_{m}(\lambda)}\right|=\left|\frac{\lambda_{-N}^{\bullet}-\lambda}{\varsigma_{N}(\lambda)}\right| \prod_{-N<m \leqslant N}\left|\frac{\lambda_{m}^{\bullet}-\lambda}{\varsigma_{m-1}(\lambda)}\right| \leqslant \frac{\left|\tau_{-N}-\lambda\right|+\left|\gamma_{-N}\right|}{|| \tau_{N}-\lambda|-| \gamma_{N}|/ 2|}
$$

By Theorem 3.1, we have $\left|\gamma_{ \pm N}\right| \leqslant \pi / 5$, as well as

$$
| \pm N-n| \pi-2 \pi / 5 \leqslant\left|\tau_{ \pm N}-\lambda\right| \leqslant| \pm N-n| \pi+2 \pi / 5 .
$$

It follows that

$$
\frac{\left|\tau_{-N}-\lambda\right|+\left|\gamma_{-N}\right|}{|| \tau_{N}-\lambda|-| \gamma_{N}|/ 2|} \leqslant \frac{|n|+N+3 / 5}{|n|-N-3 / 5} \leqslant 5\langle N\rangle
$$

Similarly, for $\lambda \in\left[\lambda_{n}^{-}, \lambda_{n}^{+}\right]$with $n<-N$.

Proposition 6.2. Suppose $\varphi \in H_{c}^{1} \cap \hat{W}$, then for any $|n| \geqslant 8\|\varphi\|_{1}^{2}$,

$$
\left|I_{n}\right| \leqslant 2^{11}\left(1+\|\varphi\|_{1}^{2}\right)\left|\gamma_{n}\right|^{2}
$$

Proof. If $\gamma_{n}=0$, then $I_{n}=0$ and the estimate clearly holds. If $\gamma_{n} \neq 0$, then by (14) and the preceding lemma,

$$
\left|4 I_{n} / \gamma_{n}^{2}\right| \leqslant 9\left|\chi_{n}\right|_{\left[\lambda_{n}^{-}, \lambda_{n}^{+}\right]} \leqslant 2^{13}\left(1+\|\varphi\|_{1}^{2}\right)
$$

Proof of Theorem 1.3. Suppose $\varphi \in H_{c}^{w} \cap \hat{W}$ and choose $N+1 \geqslant 8\|\varphi\|_{w}^{2}>N$. Then by the preceding proposition

$$
\sum_{|n|>N} w_{2 n}^{2}\left|I_{n}\right| \leqslant 2^{11}\|\varphi\|_{1}^{2} \sum_{|n|>N} w_{2 n}^{2}\left|\gamma_{n}\right|^{2}
$$


and the gap lengths may be estimated by Proposition 3.8

$$
\sum_{|n|>N} w_{2 n}^{2}\left|\gamma_{n}\right|^{2} \leqslant 6\left\|R_{N} \varphi\right\|_{w}^{2}+144\|\varphi\|_{w}^{4} \leqslant 144\left(1+\|\varphi\|_{w}^{2}\right)\|\varphi\|_{w}^{2} .
$$

In particular, the mapping

$$
H_{c}^{w} \cap \hat{W} \rightarrow[0, \infty), \quad \varphi \mapsto \sum_{n \in \mathbb{Z}} w_{2 n}^{2}\left|I_{n}\right|,
$$

is continuous. Suppose that $\varphi$ is of real type, then the remaining actions for $|n| \leqslant N$ may be estimated by

$$
\sum_{|n| \leqslant N} w_{2 n}^{2}\left|I_{n}\right| \leqslant w_{2 N}^{2} \sum_{n \in \mathbb{Z}} I_{n} \leqslant w\left[16\|\varphi\|_{w}^{2}\right]^{2}\|\varphi\|_{0}^{2}
$$

Since $w \in \mathcal{M}^{1}$ is growing with at least linear speed, we thus find on $H_{r}^{w}$

$$
\sum_{n \in \mathbb{Z}} w_{2 n}^{2}\left|I_{n}\right| \leqslant 2^{20} w\left[16\|\varphi\|_{w}^{2}\right]^{2}\|\varphi\|_{w}^{2}
$$

For any nonzero potential $\varphi$, this estimate extends by continuity to a complex neighborhood of $\varphi$ within $H_{c}^{w}$ with just the absolute constant doubled. On the other hand, sufficiently close to the zero potential we have $\|\varphi\|_{w}^{2}<\frac{1}{8}$. In this case, we may choose $N=0$ such that

$$
\sum_{n \neq 0} w_{2 n}^{2}\left|I_{n}\right| \leqslant 2^{20}\left(1+\|\varphi\|_{w}\right)^{4}\|\varphi\|_{1}^{2}
$$

Consequently, on some sufficiently small open neighborhood of $H_{r}^{w}$ in $H_{c}^{w}$,

$$
\|I(\varphi)\|_{\ell_{w}^{1}} \leqslant c_{w}^{2} w\left[16\|\varphi\|_{w}^{2}\right]^{2}\|\varphi\|_{w}^{2}
$$

with a real constant $c_{w}$ depending only on $w_{0}$.

\section{Funding}

This work was supported in part by the Swiss National Science Foundation.

\section{Acknowledgements}

The author is also very grateful to Professor Thomas Kappeler for continued support and helpful comments on this paper. 


\section{Appendix 1. Analyticity of the Primitive $F$}

In this appendix, we prove the analyticity of the primitive

$$
F(\mu, \psi)=\frac{1}{2}\left(\int_{\lambda_{0}^{-}(\psi)}^{\mu} \omega(\lambda, \psi)+\int_{\lambda_{0}^{+}(\psi)}^{\mu} \omega(\lambda, \psi)\right),
$$

introduced in Section 2 with $\omega=\frac{\Delta^{\bullet}}{\sqrt[c]{\Delta^{2}-4}} \mathrm{~d} \lambda$. The proof relies on the following observation.

Lemma A.1. (i) Suppose $\varphi \in W$ and $\gamma_{n}(\varphi)=0$ for some $n \in \mathbb{Z}$, then there exists an open neighborhood $V_{n}$ of $\varphi$ such that

$$
\left|\lambda_{n}^{\bullet}-\tau_{n}\right| \leqslant\left|\gamma_{n}\right| / 2, \quad \psi \in V_{n}
$$

(ii) For each $\varphi \in L_{r}^{2}$, there exists an open neighborhood $V \subset L_{c}^{2}$ such that

$$
\left|\lambda_{m}^{\bullet}-\tau_{m}\right| \leqslant\left|\gamma_{m}\right|, \quad m \in \mathbb{Z}, \quad \psi \in V
$$

Proof. (i) For any potential in $V_{n}=V_{\varphi}$, we have

$$
0=\left.\frac{1}{4}\left(\Delta^{2}-4\right)^{\bullet}\right|_{\lambda_{n}^{\bullet}}=\left.\left(2\left(\tau_{n}-\lambda\right) \Delta_{n}-\left(\left(\tau_{n}-\lambda\right)^{2}-\gamma_{n}^{2} / 4\right) \Delta_{n}^{\bullet}\right)\right|_{\lambda_{n}^{\bullet}},
$$

where the function $\Delta_{n}$ is analytic on $\mathbb{C} \times W$ and given by

$$
\Delta_{n}(\lambda):=\frac{1}{\pi_{n}^{2}} \prod_{m \neq n} \frac{\left(\lambda_{m}^{+}-\lambda\right)\left(\lambda_{m}^{-}-\lambda\right)}{\pi_{m}^{2}}
$$

The zeroes of $\Delta_{n}$ are precisely the eigenvalues $\lambda_{m}^{ \pm}$for $m \neq n$, thus $\Delta_{n}$ does not vanish on $U_{n} \times V_{n}$ and we have $\left|\Delta_{n}\right| \geqslant s>0$ and $\left|\Delta_{n}^{\bullet}\right| \leqslant r$ uniformly on $U_{n} \times V_{n}$. Since $\gamma_{n}(\varphi)=0$, we may shrink $V_{n}$, if necessary, to the effect that

$$
\left|\gamma_{n}(\psi)\right| r \leqslant s, \quad \psi \in V_{n}
$$

To simplify notation, put $f=2\left(\tau_{n}-\lambda\right) \Delta_{n}$ and $g=\left(\Delta^{2}-4\right)^{\bullet} / 4$. By Lemma $2.2, \gamma_{n}(\psi)=0$ implies $\lambda_{n}^{\bullet}(\psi)=\tau_{n}(\psi)$, hence we may assume $\gamma_{n}(\psi) \neq 0$. In this case, we find on the boundary of the disk $D_{\psi}=\left\{\left|\lambda-\tau_{n}(\psi)\right| \leqslant\left|\gamma_{n}(\psi)\right| / 2\right\}$,

$$
|f-g|_{\partial D_{\psi}} \leqslant\left|\gamma_{n}(\psi)\right|^{2} r / 2<s\left|\gamma_{n}(\psi)\right| \leqslant|f|_{\partial D_{\psi}} .
$$

Thus, by Rouch's Theorem, $f$ and $g$ have the same number of roots in $D_{\psi}$, and since $\lambda_{n}^{\bullet}$ is the only root of $g$ contained in $U_{n}$, the claim (i) follows. 
(ii) Recall from [10] that for each $\varphi \in L_{c}^{2}$ there exists a neighborhood $V$ and an $M \geqslant 0$ such that

$$
\sum_{|m| \geqslant M}\left|\lambda_{m}^{\bullet}-\tau_{m}\right|^{2} /\left|\gamma_{m}\right|^{4} \leqslant 1, \quad \psi \in V
$$

After possibly increasing $M$, we have $\left|\lambda_{m}^{\bullet}-\tau_{m}\right| \leqslant\left|\gamma_{m}\right|$ on $V$ for all $|m| \geqslant M$. Suppose that, in addition, $\varphi \in L_{r}^{2}$. If $\gamma_{m}(\varphi)=0$ for some $|m|<M$, then we may shrink $V$ according to (i) to obtain the desired inequality. On the other hand, if $\gamma_{m}(\varphi) \neq 0$, then

$$
\left|\lambda_{m}^{\bullet}(\varphi)-\tau_{m}(\varphi)\right| \leqslant\left|\gamma_{m}(\varphi)\right| / 2
$$

and since $\lambda_{m}^{\bullet}, \tau_{m}$, and $\left|\gamma_{m}\right|$ are continuous on $W$, the desired inequality follows after possibly shrinking $V$.

Proposition A.2. The mapping $F$ is analytic on $\left(\mathbb{C} \backslash \bigcup_{n \in \mathbb{Z}} U_{n}\right) \times V_{\varphi}$.

Proof. Take any $v \in \partial U_{0}$, and write the integral as

$$
F(\mu)=\frac{1}{2}\left(\int_{\lambda_{0}^{-}}^{\nu} \omega+\int_{\lambda_{0}^{+}}^{\nu} \omega\right)+\int_{\nu}^{\mu} \omega,
$$

where the admissible path of integration of $\int_{\lambda_{0}^{ \pm}}^{v} \omega$ runs in $U_{0} \backslash\left[\lambda_{0}^{-}, \lambda_{0}^{+}\right]$except for its end points, and the one of $\int_{v}^{\mu} \omega$ runs in $\mathbb{C} \backslash \bigcup_{n \in \mathbb{Z}} U_{n}$. Then, by Lemma 2.2, $\int_{v}^{\mu} \omega$ is analytic on $\left(\mathbb{C} \backslash \bigcup_{n \in \mathbb{Z}} U_{n}\right) \times V_{\varphi}$. We now want to prove that $F_{\nu}:=F(v)$ is analytic on $V_{\varphi}$ for an arbitrary $v \in \partial U_{0}$.

Denote by $Z_{0}:=\left\{\psi \in V_{\varphi}: \gamma_{0}^{2}(\psi)=0\right\}$ the analytic subvariety of $V_{\varphi}$ of potentials with a collapsed zeroth gap. We first prove analyticity on the open set $V_{\varphi} \backslash Z_{0}$, secondly continuity on all of $V_{\varphi}$, and finally weak analyticity on $Z_{0}$. The analyticity on $V_{\varphi}$ then follows by the general theory of analytic functions-see [14, Appendix A]. Note that by the argument above, we may move $v$ on $\partial U_{0}$ after each of the three steps.

Analyticity on $V_{\varphi} \backslash Z_{0}$. Fix any $\psi^{*}$ in $V_{\varphi} \backslash Z_{0}$. Since the eigenvalues $\lambda_{0}^{ \pm}\left(\psi^{*}\right)$ are simple, there exist two functions $\rho_{1}$ and $\rho_{2}$, which are analytic on some neighborhood $V \subset V_{\varphi} \backslash Z_{0}$ of $\psi^{*}$ and satisfy the set equality $\left\{\rho_{1}, \rho_{2}\right\}=\left\{\lambda_{0}^{-}, \lambda_{0}^{+}\right\}$. Provided the straight line $\left[\rho_{1}, \nu\right]$ is an admissible path, we can use the parameterization

$$
\lambda_{t}=\rho_{1}+t z, \quad z=v-\rho_{1},
$$


together with the product representation (4) of $\omega$ to get

$$
F_{\nu}=\int_{\rho_{1}}^{\nu} \omega=-\mathrm{i} \int_{0}^{1} \frac{\lambda_{0}^{\bullet}-\lambda_{t}}{\varsigma_{0}\left(\lambda_{t}\right)} \chi_{0}\left(\lambda_{t}\right) z \mathrm{~d} t, \quad \chi_{0}(\lambda)=\prod_{m \neq 0} \frac{\lambda_{m}^{\bullet}-\lambda}{\varsigma_{m}(\lambda)} .
$$

Note that $\chi_{0}$ is analytic on $U_{0} \times V_{\varphi}$-see [10, Section 12]. Let $\tilde{\gamma}_{0}=\rho_{2}-\rho_{1}$ and fix $v$ on $\partial U_{0}$ such that for some real $\sigma\left(\psi^{*}\right)>0$,

$$
v=\rho_{1}\left(\psi^{*}\right)-\sigma\left(\psi^{*}\right) \tilde{\gamma}_{0}\left(\psi^{*}\right) / 2 .
$$

After possibly shrinking $V$, we find for the same fixed $v$

$$
\nu=\rho_{1}(\psi)-\sigma(\psi) \tilde{\gamma}_{0}(\psi) / 2, \quad \psi \in V,
$$

with $\sigma(\psi)$ possibly complex though $\Re \sigma \geqslant \varepsilon>0$ on $V$. As $z=-\sigma \tilde{\gamma}_{0} / 2$, we conclude

$$
\left|\tau_{0}-\lambda_{t}\right|=|1+t \sigma| \cdot\left|\gamma_{0} / 2\right|>\left|\gamma_{0} / 2\right|, \quad 0<t \leqslant 1,
$$

uniformly on $V$. Therefore, the path $\left[\rho_{1}, \nu\right]$ is admissible for all $\psi$ in $V$. In view of (1), the root $\varsigma_{0}\left(\lambda_{t}\right)$ is continuous in $t \geqslant 0$, analytic on $V$ for $t>0$, and satisfies the lower bound

$$
\left|\varsigma_{0}\left(\lambda_{t}\right)\right| \geqslant\left|\gamma_{0} / 2\right| \sqrt[+]{(1+t \varepsilon)^{2}-1} \geqslant\left|\gamma_{0} / 2\right| \sqrt[+]{2 t \varepsilon}
$$

It follows that the integrand $\left(\lambda_{0}^{\bullet}-\lambda_{t}\right) \chi_{0}\left(\lambda_{t}\right) z / \varsigma_{0}\left(\lambda_{t}\right)$ of $($ A.1) is continuous on $(0,1] \times V$, analytic on $V$ for fixed $t>0$, and has an integrable majorant. In consequence, $F_{v}$ is analytic on $V$, and hence on all of $V_{\varphi} \backslash Z_{0}$.

Continuity on $V_{\varphi}$. Clearly, $F_{\nu}$ is continuous on $V_{\varphi} \backslash Z_{0}$, and its restriction

$$
\left.F_{\nu}\right|_{Z_{0}}=-\mathrm{i} \int_{\tau_{0}}^{v} \chi_{0}(\lambda) \mathrm{d} \lambda
$$

is continuous, too, as $\chi_{0}$ and $\tau_{0}$ are. To establish the continuity of $F_{v}$ on $V_{\varphi}$, it thus suffices to show that $F_{v}\left(\psi^{k}\right) \rightarrow F_{v}\left(\psi^{*}\right)$ for every sequence $\left(\psi^{k}\right)$ in $V_{\varphi} \backslash Z_{0}$ converging to some $\psi^{*}$ in $Z_{0}$.

In view of Lemma A.1, we may without loss assume $\left|\lambda_{0}^{\bullet}-\tau_{0}\right| \leqslant\left|\gamma_{0}\right| / 2$ for all $\psi^{k}$. Substituting $\lambda_{t}=\tau_{0}-t \gamma_{0} / 2$ such that $\varsigma_{0}\left(\lambda_{t}\right)^{2}=-\left(1-t^{2}\right) \gamma_{0}^{2} / 4$ gives

$$
\left|\int_{\lambda_{0}^{-}}^{\tau_{0}} \frac{\lambda_{0}^{\bullet}-\lambda}{\mathrm{i} \zeta_{0}(\lambda)} \chi_{0}(\lambda) \mathrm{d} \lambda\right| \leqslant \int_{0}^{1}\left|\frac{\lambda_{0}^{\bullet}-\tau_{0}+t \gamma_{0} / 2}{\sqrt[+]{1-t^{2}}} \chi_{0}\left(\lambda_{t}\right)\right| \mathrm{d} t=O\left(\left|\gamma_{0}\right|\right),
$$

where the implicit constant can be chosen uniformly in $k$. Therefore,

$$
F_{\nu}\left(\psi^{k}\right)=-\left.\mathrm{i} \int_{\tau_{0}}^{\nu} \frac{\lambda_{0}^{\bullet}-\lambda}{\varsigma_{0}(\lambda)} \chi_{0}(\lambda) \mathrm{d} \lambda\right|_{\psi^{k}}+o(1) .
$$


We can choose $\nu^{*}$ on $\partial U_{0}$ such that the straight line $\left[\tau_{0}, \nu^{*}\right]$ is admissible for any $\psi^{k}$. With the parameterization

$$
\lambda_{t}=\tau_{0}+t z, \quad z=v^{*}-\tau_{0}
$$

we then obtain

$$
-\mathrm{i} \int_{\tau_{0}}^{\nu^{*}} \frac{\lambda_{0}^{\bullet}-\lambda}{\varsigma_{0}(\lambda)} \chi_{0}(\lambda) \mathrm{d} \lambda=-\mathrm{i} \int_{0}^{1} \frac{\lambda_{0}^{\bullet}-\lambda_{t}}{\varsigma_{0}\left(\lambda_{t}\right)} \chi_{0}\left(\lambda_{t}\right) z \mathrm{~d} t .
$$

Since $|z|$ is uniformly bounded below on $V$,

$$
\frac{\left|\gamma_{0} / 2\right|}{\left|\tau_{0}-\lambda_{t}\right|}=O\left(\left|\gamma_{0} / t\right|\right)
$$

In view of (1) for any $\delta>0$, there exists a neighborhood $V_{\delta} \subset V$ of $\psi^{*}$ such that the root $\varsigma_{0}\left(\lambda_{t}\right)$ is continuous and does not vanish on $[\delta, 1] \times V_{\delta}$. Consequently, for all $\delta>0$,

$$
-\left.\mathrm{i} \int_{\delta}^{1} \frac{\lambda_{0}^{\bullet}-\lambda_{t}}{\varsigma_{0}\left(\lambda_{t}\right)} \chi_{0}\left(\lambda_{t}\right) z\right|_{\psi^{k}} \rightarrow-\left.\mathrm{i} \int_{\delta}^{1} \chi_{0}\left(\lambda_{t}\right) z\right|_{\psi^{*}}, \quad k \rightarrow \infty .
$$

Let $\varepsilon=\left|\gamma_{0}\right| / 2 \delta|z|$, then after possibly shrinking $V_{\delta}$ we have $0<\varepsilon<1$ and

$$
\begin{aligned}
\int_{0}^{\delta}\left|\frac{\lambda_{0}^{\bullet}-\lambda_{t}}{\varsigma_{0}\left(\lambda_{t}\right)} \chi_{0}\left(\lambda_{t}\right) z\right| \mathrm{d} t & \leqslant \int_{0}^{\delta} \frac{t|z|+\left|\gamma_{0} / 2\right|}{\sqrt{\left.\left|t^{2}\right| z\right|^{2}-\left|\gamma_{0} / 2\right|^{2} \mid}}\left|\chi_{0}\left(\lambda_{t}\right) z\right| \mathrm{d} t \\
& =O\left(\delta \int_{0}^{1} \frac{\sqrt[+]{|t+\varepsilon|}}{\sqrt[+]{|t-\varepsilon|}} \mathrm{d} t\right)
\end{aligned}
$$

where the implicit constant is uniform on $V_{\delta}$. One checks that the latter integral is uniformly bounded for $0 \leqslant \varepsilon \leqslant 1$, hence

$$
\sup _{\psi \in V_{\delta}} \int_{0}^{\delta}\left|\frac{\lambda_{0}^{\bullet}-\lambda_{t}}{\varsigma_{0}\left(\lambda_{t}\right)} \chi_{0}\left(\lambda_{t}\right) z\right| \mathrm{d} t=O(\delta) .
$$

It follows that

$$
-\left.\mathrm{i} \int_{0}^{1} \frac{\lambda_{0}^{\bullet}-\lambda_{t}}{\varsigma_{0}\left(\lambda_{t}\right)} \chi_{0}\left(\lambda_{t}\right) z\right|_{\psi^{k}} \rightarrow-\left.\mathrm{i} \int_{0}^{1} \chi_{0}\left(\lambda_{t}\right) z\right|_{\psi^{*}}, \quad k \rightarrow \infty,
$$

so $F_{v^{*}}$ is continuous at $\psi^{*}$ and hence on all of $V_{\varphi}$.

Weak analyticity. The restriction of $F_{\nu}$ to $Z_{0}$ is given by

$$
\left.F_{\nu}\right|_{z_{0}}=-\mathrm{i} \int_{\tau_{0}}^{\nu} \chi_{0}(\lambda) \mathrm{d} \lambda
$$

and since $\chi_{0}$ and $\tau_{0}$ are both analytic, the weak analyticity follows directly. This completes the proof of the analyticity of $F_{\nu}$ on $V_{\varphi}$. 


\section{Appendix 2. Properties of the NLS Hierarchy}

In this appendix, we recall some well-known facts about the Hamiltonians of the NLS hierarchy-see [10]. For $\varphi=\left(\varphi_{-}, \varphi_{+}\right) \in H_{c}^{k-1}$, the $k$ th NLS Hamiltonian is given by

$$
\mathcal{H}_{k}(\varphi)=\int_{\mathbb{T}} \varphi_{-} u_{k}\left(\varphi_{-}, \varphi_{+}, \ldots, \varphi_{-}^{(k-1)}, \varphi_{+}^{(k-1)}\right) \mathrm{d} x, \quad k \geqslant 1
$$

where $u_{1}=-\varphi_{+}$, and

$$
u_{k+1}=u_{k}^{\prime}+\varphi_{-} \sum_{l=1}^{k-1} u_{k-l} u_{l}, \quad k \geqslant 1
$$

Lemma A.3. If $\varphi \in H_{c}^{k-1}$, then

$$
u_{k+1}=-\varphi_{+}^{(k)}+q_{k}\left(\varphi_{-}, \varphi_{+}, \ldots, \varphi_{-}^{(k-2)}, \varphi_{+}^{(k-2)}\right)
$$

where $q_{k}$ is a homogeneous polynomial of degree $k+1$ when $\varphi_{-}, \varphi_{+}$, and $\partial_{X}$ each count as one degree. Moreover, each term of $\varphi_{-} q_{k}$ has at most degree $k-2$ with respect to $\partial_{x}$, and the degree with respect to $\varphi_{-}$equals the one with respect to $\varphi_{+}$.

Proof. As is evident from their definition, the polynomials $u_{k}$ are homogeneous of degree $k$, and only contain derivatives of $\varphi_{-}$and $\varphi_{+}$up to order $k-1$. Furthermore, $u_{k}\left(\varphi_{-}, \lambda \varphi_{+}\right)=\lambda u_{k}\left(\lambda \varphi_{-}, \varphi_{+}\right)$for all $\lambda \in \mathbb{C}$, which completes the proof.

In case of a smooth real-type potential, that is, $\varphi=(\psi, \bar{\psi})$, the odd Hamiltonians have the form

$$
(-1)^{m+1} \mathcal{H}_{2 m+1}(\varphi)=\int_{\mathbb{T}}\left(\left|\psi^{(m)}\right|^{2}+\psi q_{2 m}\right) \mathrm{d} x, \quad m \geqslant 1,
$$

where $q_{2 m}$ depends on $\psi, \bar{\psi}$, and their derivatives up to order $2 m-2$. Suppose that $\psi q_{2 m}$ contains a monomial $\psi_{(m+n)} \mathfrak{q}\left(\psi, \bar{\psi}, \ldots, \psi_{(2 m-2)}, \bar{\psi}_{(2 m-2)}\right)$ with $n \geqslant 0$. Since this monomial has at most degree $2 m-2$ with respect to $\partial_{X}$, it follows that $\mathfrak{q}$ contains at most $m-2-n$ derivatives. Thus, we can integrate by parts to the effect that each factor of the monomial contains at most $m-1$ derivatives.

Corollary A.4. For any $m \geqslant 1$, there exists a polynomial $p_{2 m}$ such that

$$
(-1)^{m+1} \mathcal{H}_{2 m+1}(\varphi)=\int_{\mathbb{T}}\left(\left|\psi_{(m)}\right|^{2}+p_{2 m}\left(\psi, \bar{\psi}, \ldots, \psi_{(m-1)}, \bar{\psi}_{(m-1)}\right)\right) \mathrm{d} x
$$


for all $\varphi=(\psi, \bar{\psi})$ in $H_{r}^{m}$. The polynomial $p_{2 m}$ is homogenous of degree $2 m+2$ with respect to $\psi, \bar{\psi}$, and $\partial_{X}$, and the degree of each term of $p_{2 m}$ with respect to $\psi$ equals the one with respect to $\bar{\psi}$.

\section{References}

[1] Bättig, D., A. M. Bloch, J. C. Guillot, and T. Kappeler. "On the symplectic structure of the phase space for periodic KdV, Toda, and defocusing NLS." Duke Mathematical Journal 79, no. 3 (1995): 549-604.

[2] Bättig, D., B. Grébert, J. C. Guillot, and T. Kappeler. "Foliation of phase space for the cubic nonlinear Schrödinger equation." Compositio Mathematica 85, no. 2 (1993): 163-99.

[3] Colliander, J., M. Keel, G. Staffilani, H. Takaoka, and T. Tao. “Global well-posedness for KdV in Sobolev spaces of negative index." Electronic Journal of Differential Equations 2001, no. 26 (2001): 1-7 (electronic).

[4] Colliander, J., M. Keel, G. Staffilani, H. Takaoka, and T. Tao. “Global well-posedness for Schrödinger equations with derivative." SIAM Journal on Mathematical Analysis 33, no. 3 (2001): 649-69 (electronic).

[5] Colliander, J., M. Keel, G. Staffilani, H. Takaoka, and T. Tao. “Multilinear estimates for periodic KdV equations, and applications." Journal of Functional Analysis 211, no. 1 (2004): 173-218.

[6] Colliander, J., M. Keel, G. Staffilani, H. Takaoka, and T. Tao. “Transfer of energy to high frequencies in the cubic defocusing nonlinear Schrödinger equation." Inventiones Mathematicae 181, no. 1 (2010): 39-113.

[7] Djakov, P. and B. Mityagin. "Instability zones of periodic 1-dimensional Schrödinger and Dirac operators." Russian Mathematical Surveys 61, no. 4 (2006): 663-766.

[8] Flaschka, H. and D. W. McLaughlin. "Canonically conjugate variables for the Kortewegde Vries equation and the Toda lattice with periodic boundary conditions." Progress of Theoretical Physics 55, no. 2 (1976): 438-56.

[9] Grébert, B. and T. Kappeler. "Estimates on periodic and Dirichlet eigenvalues for the Zakharov-Shabat system." Asymptotic Analysis 25, no. 3-4 (2001): 201-37.

[10] Grébert, B. and T. Kappeler. The Defocusing NLS Equation and its Normal Form. EMS Series of Lectures in Mathematics, $\mathrm{x}+166$ pp. Zürich: European Mathematical Society (EMS), 2014.

[11] Grébert, B., T. Kappeler, and B. Mityagin. “Gap estimates of the spectrum of the ZakharovShabat system." Applied Mathematics Letters 11, no. 4 (1998): 95-7.

[12] Kappeler, T. and B. Mityagin. "Gap estimates of the spectrum of Hill's equation and action variables for KdV." Transactions of the American Mathematical Society 351, no. 2 (1999): 619-46.

[13] Kappeler, T. and B. Mityagin. "Estimates for periodic and Dirichlet eigenvalues of the Schrödinger operator." SIAM Journal on Mathematical Analysis 33, no. 1 (2001): 113-52 (electronic).

[14] Kappeler, T. and J. Pöschel. KdV \& KAM, vol. 45. Berlin: Springer, 2003. 
[15] Kappeler, T. and J. Pöschel. "On the periodic KdV equation in weighted Sobolev spaces." Annales de l'Institut Henri Poincaré. Analyse Non Linéaire 26, no. 3 (2009): 841-53.

[16] Kappeler, T., F. Serier, and P. Topalov. "On the characterization of the smoothness of skewadjoint potentials in periodic Dirac operators." Journal of Functional Analysis 256, no. 7 (2009): 2069-112.

[17] Kappeler, T., P. Topalov, and B. Schaad. "Asymptotics of spectral quantities of ZhakarovShabat operators." (in preparation).

[18] Korotyaev, E. “Estimates for the Hill operator. I." Journal of Differential Equations 162, no. 1 (2000): 1-26.

[19] Korotyaev, E. "Inverse problem and estimates for periodic Zakharov-Shabat systems." Journal für die Reine und Angewandte Mathematik 2005, no. 583 (2005): 87-115.

[20] Korotyaev, E. "Estimates for the Hill operator. II." Journal of Differential Equations 223, no. 2 (2006): 229-60.

[21] Korotyaev, E. and P. Kargaev. "Estimates for periodic Zakharov-Shabat operators." Journal of Differential Equations 249, no. 1 (2010): 76-93.

[22] Li, Y. and D. W. McLaughlin. "Morse and Mel'nikov functions for NLS PDEs." Communications in Mathematical Physics 162, no. 1 (1994): 175-214.

[23] McKean, H. P. and K. L. Vaninsky. "Action-angle variables for the cubic Schrödinger equation." Communications on Pure and Applied Mathematics 50, no. 6 (1997): 489-562.

[24] Mityagin, B. "Spectral expansions of one-dimensional periodic Dirac operators." Dynamics of Partial Differential Equations 1, no. 2 (2004): 125-91.

[25] Molnar, J. C. "Uniform estimates of the nonlinear Fourier transform for the KdV equation." (in preparation).

[26] Pöschel, J. "Hill's potentials in weighted Sobolev spaces and their spectral gaps." Mathematische Annalen 349, no. 2 (2011): 433-58.

[27] Zakharov, V. E. and A. B. Shabat. "A scheme for integrating the nonlinear equations of mathematical physics by the method of the inverse scattering problem. I." Functional Analysis and its Applications 8, no. 3 (1975): 226-35. 